\title{
Evolution of the Systems Pharmacokinetics-Pharmacodynamics Model for Antibody-Drug Conjugates to Characterize Tumor Heterogeneity and In Vivo Bystander Effect ${ }^{[}$
}

\author{
Aman P. Singh, (1) Gail M. Seigel, Leiming Guo, Ashwni Verma, Gloria Gao-Li Wong, \\ Hsuan-Ping Cheng, and Dhaval K. Shah
}

Department of Pharmaceutical Sciences, School of Pharmacy and Pharmaceutical Sciences (A.P.S., L.G., A.V., H.-P.C., D.K.S.), Center for Hearing and Deafness, SUNY Eye Institute (G.M.S.), and Department of Biological Sciences (G.G.-L.W.), The State University of New York at Buffalo, Buffalo, New York

Received August 24, 2019; accepted March 30, 2020

\begin{abstract}
The objective of this work was to develop a systems pharmacokinetics-pharmacodynamics (PK-PD) model that can characterize in vivo bystander effect of antibody-drug conjugate $(A D C)$ in a heterogeneous tumor. To accomplish this goal, a coculture xenograft tumor with 50\% GFP-MCF7 (HER2-low) and 50\% N87 (HER2-high) cells was developed. The relative composition of a heterogeneous tumor for each cell type was experimentally determined by immunohistochemistry analysis. Trastuzumab-vc-MMAE (T-vc-MMAE) was used as a tool ADC. Plasma and tumor PK of T-vc-MMAE was analyzed in N87, GFP-MCF7, and coculture tumorbearing mice. In addition, tumor growth inhibition (TGI) studies were conducted in all three xenografts at different T-vc-MMAE dose levels. To characterize the PK of ADC in coculture tumors, our previously published tumor distribution model was evolved to account for different cell populations. The evolved tumor PK model was able to a priori predict the PK of all ADC analytes in the coculture tumors reasonably well. The tumor PK model was subsequently integrated with a PD model that used intracellular tubulin occupancy to drive ADC efficacy
\end{abstract}

in each cell type. The final systems PK-PD model was able to simultaneously characterize all the TGI data reasonably well, with a common set of parameters for MMAE-induced cytotoxicity. The model was later used to simulate the effect of different dosing regimens and tumor compositions on the bystander effect of ADC. The model simulations suggested that dose-fractionation regimen may further improve overall efficacy and bystander effect of ADCs by prolonging the tubulin occupancy in each cell type.

\section{SIGNIFICANCE STATEMENT}

A PK-PD analysis is presented to understand bystander effect of Trastuzumab-vc-MMAE ADC in antigen (Ag)-low, Ag-high, and coculture (i.e., Ag-high + Ag-low) xenograft mice. This study also describes a novel single cell-level systems PK-PD model to characterize in vivo bystander effect of ADCs. The proposed model can serve as a platform to mathematically characterize multiple cell populations and their interactions in tumor tissues. Our analysis also suggests that fractionated dosing regimen may help improve the bystander effect of ADCs.

\section{Introduction}

Antibody-drug conjugates (ADCs) are novel anticancer drug molecules (Lambert, 2015; Mantaj et al., 2017), which are designed to expand the therapeutic window of chemotherapeutic agents by targeting them to antigen expressing tumor cells via monoclonal antibodies. At present, there are five Food and Drug Administration-approved ADCs and $>80$ molecules in the clinical development (Anthony, 2018). Though these

This work was supported by the Centre for Protein Therapeutics at University at Buffalo. D.K.S. is supported by National Institutes of Health National Institute of General Medical Sciences [Grant R01-GM114179] and National Institute of Allergy and Infectious Diseases [Grant R21-AI138195].

The authors declare no conflict of interest.

https://doi.org/10.1124/jpet.119.262287.

S This article has supplemental material available at jpet.aspetjournals.org. molecules are promising, their efficacy is often limited by poor tumor distribution (Cilliers et al., 2016) and heterogeneity in antigen (Ag)-expressing (Ag+) cells (Seol et al., 2012). These limitations can be overcome with the help of "bystander effect," which promotes more homogenous distribution of payload within a solid tumor (Kovtun et al., 2006; Singh et al., 2016b). Once the ADC molecules bind to Ag+ cells in a heterogeneous tumor, they get internalized and release the payload, a fraction of which kills the Ag+ cells, and the rest diffuse out and kill the bystanding antigen-negative $(\mathrm{Ag}-)$ cells, leading to a phenomenon known as the bystander effect (Singh et al., 2016b). Though this phenomenon is qualitatively confirmed using in vitro and in vivo investigations (Kovtun et al., 2006; Ogitani et al., 2016), the rate and extent of bystander effect within a heterogeneous tumor is not

ABBREVIATIONS: ADC, antibody-drug conjugate; Ag, antigen; DAPI, 4',6-Diamidino-2-Phenylindole; DAR, drug:antibody ratio; GoF, goodness of fit; HER2, human epidermal growth factor receptor 2; IHC, immunohistochemical; MMAE, monomethyl auristatin E; PK-PD, pharmacokineticspharmacodynamics; SCID, severe combined immunodeficient; TGI, tumor growth inhibition; Tmax, time taken to achieve maximum exposure; T-vcMMAE, Trastuzumab-vc-MMAE; vc, valine-citrulline. 
quantitatively determined or mathematically characterized to date. To further enhance quantitative understanding of this phenomenon, here we present an in vivo investigation and development of a systems pharmacokinetic-pharmacodynamic (PK-PD) model to characterize in vivo bystander effect of ADCs. Development of such models can be crucial for identifying drugand system-specific parameters that are important for accomplishing in vivo bystander effect (Singh et al., 2015a,b; Singh and Shah, 2017a, 2018). In addition, these models could also be used to guide the development of next-generation ADCs (van Geel et al., 2015), evaluate druggability of novel targets, and facilitate preclinical-to-clinical translation of ADC efficacy.

In the past, we have presented quantitative investigation to understand in vitro bystander effect of ADCs using anti-HER2 ADC Trastuzumab-vc-MMAE (T-vc-MMAE) as a tool compound. Upon internalization, this ADC can release the lipophilic payload MMAE in its pure form, which is capable of partitioning into the nearby cells, and exhibit bystander killing. Using in vitro cocultures of HER2-high N87 (Ag+) and HER2-low GFP-MCF7 (Ag-) cells, we have investigated rate and extent of ADC-induced bystander effect and evaluated how the presence of different percentage of $\mathrm{Ag}+$ cells affect the bystander killing of Ag- cells (Singh et al., 2016b). During these investigations, we observed that there was a notable delay between $\mathrm{ADC}$ exposure and the occurrence of bystander effect, which may have stemmed from the time taken by the cells to process the ADCs. Consequently, we further investigated cellular processing of T-vc-MMAE using in vitro PK studies. With the help of the PK data, we were able to develop a unique single-cell PK model for ADCs, which was able to integrate key drug- and system-specific parameters in a mathematical format to characterize ADC processing within different cell lines (Singh and Shah, 2017b). We further expanded this single-cell PK model to develop an in vitro systems PK-PD model, which employed intracellular occupancy of the pharmacological target (i.e., tubulin) by MMAE to drive direct and bystander killing of cancer cells by the ADC (Singh and Shah, 2019). This unique single-cell PKPD model has also been translated to in vivo scenarios recently to characterize tumor $\mathrm{PK}$ of T-vc-MMAE and tumor growth inhibition (TGI) by T-vc-MMAE in GFP-MCF7 and N87 xenografts (Singh et al., 2019).

Here, we have expanded the application of cell-level PK-PD model toward characterizing in vivo bystander effect for T-vcMMAE in a heterogeneous tumor model developed using GFPMCF7 and N87 cells. Both the cell lines were inoculated in a mouse to develop a 50:50 coculture xenograft tumor model. PK and PD (i.e., TGI) of T-vc-MMAE was investigated in the coculture xenograft. To quantitatively characterize tumor PK of ADC, our previously published tumor disposition model was evolved to account for tumor heterogeneity. The model also accounted for interaction of T-vc-MMAE and released MMAE with two different cell populations in the tumor and distinct growth characteristics of each cell population. The evolved model was able to a priori predict tumor PK of T-vc-MMAE in the heterogeneous tumor model. Subsequently, the tumor PK model was integrated with our previously published singlecell PK-PD model to characterize T-vc-MMAE-induced direct and bystander killing of cancer cells in the coculture tumor. The final systems PK-PD model was used to evaluate the effect of different dosing regimen and tumor composition on the bystander effect of ADC. The presented PK-PD model could be translated to predict the bystander effect of ADCs in the clinic using the strategies presented by us previously (Shah et al., 2012; Singh and Shah, 2017a).

\section{Materials and Methods}

\section{Cell Lines}

HER2-expressing cell lines NCI-N87 and GFP-transfected MCF7 were cultured using their recommended media conditions (Singh et al., 2016b; Singh and Shah, 2017b). HER2 expression level in N87 and GFP-MCF7 cells has been reported to be $\sim 950,000$ and $\sim 55,000$ receptors per cell, which makes them HER2-high and HER2-low cell lines, respectively (Singh and Shah, 2017b). Expression of GFP in MCF7 cells enables their detection within in vitro and in vivo coculture system (Singh et al., 2016b).

\section{Tool ADC}

The tool ADC T-vc-MMAE was synthesized and characterized inhouse. Commercially available Trastuzumab (Herceptin; Genentech) was conjugated with valine-citrulline MMAE (drug-linker solution) using random conjugation method, resulting in a heterogeneous formulation with an average drug:antibody ratio (DAR) of $\sim 4$. Detailed protocols on synthesis and characterization of T-vc-MMAE have been published before (Singh et al., 2016b; Singh and Shah, 2017b).

\section{Development of N87 and GFP-MCF7 Monoculture Xenografts}

Six-week-old male severe combined immunodeficient (SCID) (NOD.CB17-Prkdc ${ }^{\text {scid }} / J$ ) mice were purchased from Jackson Laboratory. After acclimation, mice were subcutaneously implanted with $\sim 10$ million tumor cells (N87 or GFP-MCF7) suspended in their growth medium. To facilitate faster growth of GFP-MCF7-bearing tumors, mice were supplemented with $1 \mu \mathrm{g} 17 \beta$-estradiol valerate (Sigma) in $50 \mu \mathrm{l}$ pharmaceutical-grade peanut oil (Sigma) via subcutaneous injection every 4 days (Donoghue et al., 2012). All procedures were approved by the Institutional Animal Care and Use Committee at State University of New York at Buffalo.

\section{Development of N87 and GFP-MCF7 Coculture Xenografts}

Because of variable growth patterns of $\mathrm{N} 87$ and GFP-MCF7 cells, different ratios of two cell types were initially implanted to find out a ratio that would yield 50:50 coculture xenograft at the time of ADC administration. After optimization, a mixture of $\sim 1$ million GFPMCF7 and $\sim 9$ million N87 cells suspended in a similar ratio (1:9) of their cell culture medium was used to achieve 50:50 coculture at tumor volumes $\sim 350-400 \mathrm{~mm}^{3}$. To enable successful growth of GFPMCF7 cells, and to maintain consistency, mice were also supplemented with subcutaneous dosing of $17 \beta$-estradiol valerate as mentioned before.

\section{Immunohistochemical Analysis of Heterogeneous Tumors}

Heterogeneous tumors were grown in SCID mice by inoculating $\sim 1$ million GFP-MCF7 and $~ 9$ million N87 cells, as described earlier. Once the tumors reached a volume of $350-400 \mathrm{~mm}^{3}$, mice were sacrificed, and tumors were surgically removed. The harvested tissues were fixed in $4 \%$ paraformaldehyde for 24 hours and were later transferred to $70 \%$ ethanol. The tissues were then embedded in paraffin, and $4-\mu \mathrm{m}$-thick sections were cut using a microtome from an upper, middle, and lower part of a spherical tumor. The obtained sections were rinsed in PBS (PH 7.4) twice and later stained using 4',6Diamidino-2-Phenylindole (DAPI) (Sigma) at a working concentration of $1 \mu \mathrm{g} / \mathrm{ml}$ for 5 minutes at room temperature in the dark. The stained slides were imaged using Zeiss AxioImager upright microscope to measure the fluorescence emitted from GFP label (excitation: $488 \mathrm{~nm}$; 
emission: $510 \mathrm{~nm}$ ) and DAPI (excitation: $360 \mathrm{~nm}$; emission: $460 \mathrm{~nm}$ ) counterstain. To perform semiquantitative analysis of immunohistochemical (IHC) images, the images obtained from the middle sections of coculture tumors $(n=3)$ were analyzed using ImageJ software (Schneider et al., 2012). Percentage of areas emitting fluorescence from GFP label and DAPI counterstain were calculated. Because DAPI will stain the nuclei of all tumor cells, the percentage of a given section emitting blue fluorescence from DAPI will reflect the fraction of tumor area covered with both cell types (i.e., N87 or GFP-MCF7). However, the percentage of a given section emitting green fluorescence from GFP will reflect the fraction of tumor area covered with only GFP-MCF7 cells. The ratio of these two measurements should provide a rough estimate of the fraction of each cell-type within a heterogeneous tumor.)

\section{Bioanalytical Techniques to Measure Plasma and Tumor Concentrations of ADC}

Three different analytical methods were developed to measure plasma and tumor PK of T-vc-MMAE. An enzyme-linked immunosorbent assay method was used to measure total Trastuzumab concentrations, whereas a liquid chromatography-tandem mass spectrometry method was used to measure free MMAE concentrations in plasma and tumor homogenate samples. A forced deconjugation method using papain enzyme was employed to deconjugate the antibody-conjugated MMAE in a biologic sample, which enabled the measurement of total MMAE in plasma and tumor samples. Detailed methodology for the development and validation of these analytical techniques (Singh and Shah, 2017b), and their application for in vitro and in vivo experiments (Singh et al., 2019), have been published before.

\section{Plasma and Tumor Pharmacokinetic Studies}

A total of 30 SCID mice were equally divided into three groups, which were then inoculated with GFP-MCF7 (group A, $n=10$ ), N87 cells (group B, $n=10$ ), or 1:9 mixture of GFP-MCF7 and N87 cells (group C, $n=10$ ). Treatment was initiated $3-6$ weeks after implantation when the tumors were $\sim 400 \mathrm{~mm}^{3}$. Nine out of 10 animals from each group (A, B, or C) were treated with a single dose of $10 \mathrm{mg} / \mathrm{kg}$ T-vc-MMAE intravenously, whereas one untreated animal from each group was sacrificed to harvest blood and tumor samples on day 0 . Treated animals were further divided into three different subgroups $(n=3)$ corresponding to the 24-hour (A1, B1, C1), 72-hour (A2, B2, C2), and 168-hour (A3, B3, C3) time points. At each time point, blood samples were collected via cardiac puncture, and tumor tissues were surgically removed. In addition, a blood sample was collected from one animal in each subgroup $(n=3)$ at 10 minutes. Harvested blood samples were immediately processed to separate plasma, and all plasma and tumor samples were stored at $-80^{\circ} \mathrm{C}$ until further analysis.

\section{Tumor Growth Inhibition Studies}

Single-Dose Therapy of HER2-High N87 Xenografts with T-ve-MMAE. A total of 28 SCID mice were subcutaneously inoculated with N87 cells, and the treatment was initiated 4-6 weeks after tumor implantation when the tumor volumes reached $\sim 400 \mathrm{~mm}^{3}$. At day 0 , mice were randomly assigned to four equal groups: control (no treatment, $n=7), 1 \mathrm{mg} / \mathrm{kg}$ T-vc-MMAE ( $n=7), 3 \mathrm{mg} / \mathrm{kg}$ T-vc-MMAE $(n=7)$, and $10 \mathrm{mg} / \mathrm{kg} \mathrm{T}-\mathrm{vc}-\mathrm{MMAE}$.

Single-Dose Therapy of HER2-Low GFP-MCF7 Xenografts with T-vc-MMAE. A total of 25 SCID mice were subcutaneously inoculated with GFP-MCF7 cells, and the treatment was initiated 3-5 weeks after tumor implantation when the tumor volumes reached $\sim 400 \mathrm{~mm}^{3}$. At day 0, mice were randomly assigned to four different groups: control (no treatment, $n=6), 3 \mathrm{mg} / \mathrm{kg} \mathrm{T-vc-MMAE}(n=7)$, $5 \mathrm{mg} / \mathrm{kg}$ T-vc-MMAE $(n=5)$, and $10 \mathrm{mg} / \mathrm{kg}$ T-vc-MMAE $(n=7)$.
Single-Dose Therapy of 50:50 Coculture Xenografts of N87 and GFP-MCF7 Cells with T-ve-MMAE. A total of 20 SCID mice were subcutaneously inoculated with a mixture of GFP-MCF7 and N87 cells (ratio 1:9), and the treatment was initiated 3-5 weeks after tumor implantation when the tumor volumes reached $\sim 400 \mathrm{~mm}^{3}$. At day 0 , mice were randomly assigned to four equal groups: control (no treatment, $n=5$ ), $3 \mathrm{mg} / \mathrm{kg}$ T-vc-MMAE $(n=5), 5 \mathrm{mg} / \mathrm{kg}$ T-vc-MMAE $(n=5)$, and $10 \mathrm{mg} / \mathrm{kg} \mathrm{T-vc-MMAE}(n=5)$.

Tumor volumes were calculated by measuring length (L, distance between longest diameter) and breadth (B) of tumors using vernier caliper and the following formula: $\frac{1}{2} \times \mathrm{L} \times \mathrm{B}^{2}$. Tumor volumes were measured twice a week until the tumor volume exceeded $>2500 \mathrm{~mm}^{3}$ cutoff or the tumor was completely regressed for a prolonged duration of time ( $\sim 3$ weeks).

\section{Development of the Systems PK-PD Model to Characterize In Vivo Bystander Effect of Trastuzumab-vc-MMAE}

Plasma Pharmacokinetic Model for T-ve-MMAE. A previously established plasma PK model for ADCs (shown as a part of Fig. 3) was used to build this model. Because plasma PK of ADC was similar between N87, GFP-MCF7, and coculture xenografts, plasma PK data from all three mouse models were pooled to estimate the model parameters. Briefly, the biexponential PK profile of ADC is characterized using a two-compartment model with linear catabolic clearance $\left(\mathrm{CL}_{\mathrm{ADC}}\right)$ from the central compartment. The distribution of $\mathrm{ADC}$ to peripheral tissues is characterized using a distributional clearance parameter $\left(\mathrm{CLD}_{\mathrm{ADC}}\right)$. An additional first-order elimination rate constant coined as "nonspecific deconjugation rate" $\left(\mathrm{K}_{\mathrm{dec}}^{\mathrm{P}}\right)$ is incorporated in the central compartment to characterize nonspecific deconjugation of MMAE from the ADC. This rate also contributes toward the first-order decline in average DAR value of the ADC. Both elimination pathways $\left(\mathrm{CL}_{\mathrm{ADC}}\right.$ and $\mathrm{K}_{\mathrm{dec}}^{\mathrm{P}}$ ) generate free (unconjugated) MMAE and hence become the formation rates for MMAE. The disposition of free MMAE is also characterized using a twocompartment model with linear systemic clearance $\left(\mathrm{CL}_{\text {Drug }}\right)$ and distributional clearance $\left(\mathrm{CLD}_{\text {Drug }}\right)$ (Shah et al., 2012, 2014; Khot et al., 2015; Singh et al., 2015b, 2016a; Singh and Shah, 2017a, 2018).

Tumor Distribution Model for T-vc-MMAE. Distribution of T-vc-MMAE and released MMAE in solid tumors was characterized using the tumor disposition model shown in Fig. 3. Two distinct exchange processes (i.e., surface and vascular exchange) are incorporated to describe the mechanistic distribution of T-vc-MMAE and free MMAE from systemic circulation to tumor extracellular space. Because of high interstitial pressure and lack of functional lymphatic system within the tumor microenvironment, it is assumed that the disposition of ADC and released drug is limited to diffusive processes, with a lack of any convective transport. Within the original work published by Wittrup et al. (Thurber et al., 2008a,b), molecular sizespecific diffusivity and permeability parameters have been reported. Additionally, with an assumption of a spherical tumor, size/radius of the tumor was used to determine the rate of $\mathrm{ADC} / \mathrm{released}$ drug exchange via surface and vascular processes. At lower tumor volumes, surface exchange predominates, and at higher tumor volumes, vascular exchange is more predominant (Thurber et al., 2008a,b). With changing tumor volumes because of $\mathrm{ADC}$ efficacy, the relative significance of these pathways also varies.

Incorporation of Single-Cell Pharmacokinetic Model to Account for ADC Processing by N87 and GFP-MCF7 Cells. Once in the tumor extracellular space, the disposition of T-vc-MMAE and released MMAE within each cell type (N87 or GFP-MCF7) was characterized using a single-cell disposition model (Fig. 3) (Singh and Shah, $2017 \mathrm{~b}$ ). It was assumed that there are $\sim 10^{8}$ tumor cells per gram of tumor (Del Monte, 2009). Exchange of T-vc-MMAE and free MMAE between each cell type and extracellular space was characterized simultaneously. Key processes in the model include T-vc-MMAE binding $\left(\mathrm{K}_{\mathrm{on}}^{\mathrm{ADC}}\right.$ and $\left.\mathrm{K}_{\mathrm{off}}^{\mathrm{ADC}}\right)$ to HER2 receptors on the tumor cells and internalization $\left(\mathrm{K}_{\mathrm{int}}^{\mathrm{ADC}}\right)$ and intracellular degradation $\left(\mathrm{K}_{\mathrm{deg}}^{\mathrm{ADC}}\right)$ of $\mathrm{ADC}$ in 
the endosomal/lysosomal space, which leads to the release of the free (unconjugated) MMAE in the cytoplasm. The released MMAE within the cytoplasmic space is assumed to either bind to intracellular tubulin $\left(\mathrm{K}_{\mathrm{on}}^{\mathrm{Tub}}\right.$ and $\mathrm{K}_{\mathrm{off}}^{\mathrm{Tub}}$ ) or exchange with the extracellular space using influx $\left(\mathrm{K}_{\mathrm{in}}^{\text {Drug }}\right)$ and efflux $\left(\mathrm{K}_{\mathrm{out}}^{\text {Drug }}\right)$ processes. Once the free MMAE is released in the tumor extracellular space, it can either distribute to other cancer cells or diffuse out into the systemic circulation.

To conserve mass balance within the dynamic system of two distinctly growing tumor cell populations, a dilution factor was incorporated within the single-cell PK model for each cell type. This dilution factor renders dilution of intracellular content (either intact $\mathrm{ADC}$ or released drug) at the rate equivalent to the growth rate $\left(\mathrm{K}_{\mathrm{g}}^{\mathrm{Tumor}}\right)$ of each cell type.

Equations pertaining to the growth of each cell type (i.e., N87 or GFPMCF7) within the heterogeneous tumor (Tumor volume(TV), in L) are provided below. A 50:50 coculture composition was used as the initial condition:

$$
\begin{gathered}
\frac{\mathrm{d}\left(\mathrm{TV}_{\mathrm{mm} 3}^{\mathrm{N} 87}\right)}{\mathrm{dt}}=\frac{\mathrm{Ln} 2}{\mathrm{DT}^{\mathrm{N} 87}} \cdot \mathrm{TV}_{\mathrm{mm} 3}^{\mathrm{N} 87} \quad \text { Initial Condition }(\mathrm{IC})=\frac{1}{2} \cdot \mathrm{TV}_{\mathrm{mm} 3}^{0} \\
\frac{\mathrm{d}\left(\mathrm{TV}_{\mathrm{mm} 3}^{\mathrm{MCF7}}\right)}{\mathrm{dt}}=\frac{\mathrm{Ln} 2}{\mathrm{DT}^{\mathrm{MCF} 7}} \cdot \mathrm{TV}_{\mathrm{mm} 3}^{\mathrm{MCF} 7} \quad \mathrm{IC}=\frac{1}{2} \cdot \mathrm{TV}_{\mathrm{mm} 3}^{0} \\
\mathrm{TV}_{\mathrm{mm} 3}=\mathrm{TV}_{\mathrm{mm} 3}^{\mathrm{N} 87}+\mathrm{TV}_{\mathrm{mm} 3}^{\mathrm{MCF} 7} \\
\mathrm{TV}_{\mathrm{mm} 3}=\mathrm{TV} \cdot 10^{6} \quad \mathrm{R}_{\mathrm{Tumor}}=\left[\frac{3 \cdot \mathrm{TV} \cdot 1000}{4 \pi}\right]^{1 / 3} .
\end{gathered}
$$

Above, $\mathrm{TV}_{\mathrm{mm} 3}^{0}$ corresponds to initial tumor volume of the heterogenous tumor at the beginning of the treatment.

Equations associated with plasma pharmacokinetics and tumor distribution of T-vc-MMAE (in amounts) and unconjugated MMAE (in concentrations) are provided below:

$$
\begin{aligned}
& \frac{\mathrm{d}\left(\mathrm{X} 1_{\mathrm{ADC}}\right)}{\mathrm{dt}}=-\frac{\mathrm{CL}_{\mathrm{ADC}}}{\mathrm{V} 1_{\mathrm{ADC}}} \cdot \mathrm{X} 1_{\mathrm{ADC}}-\frac{\mathrm{CLD}_{\mathrm{ADC}}}{\mathrm{V} 1_{\mathrm{ADC}}} \cdot \mathrm{X} 1_{\mathrm{ADC}}+\frac{\mathrm{CLD} \mathrm{ADC}_{\mathrm{ADC}}}{\mathrm{V} 2_{\mathrm{ADC}}} \\
& \cdot \mathrm{X} 2_{\mathrm{ADC}}-\mathrm{K}_{\mathrm{dec}}^{\mathrm{P}} \\
& \cdot \mathrm{X} 1_{\mathrm{ADC}}-\left(\frac{\mathrm{X} 1_{\mathrm{ADC}}}{\mathrm{V} 1_{\mathrm{ADC}}}-\frac{\mathrm{ADC}_{\mathrm{f}}^{\mathrm{ex}}}{\varepsilon^{\mathrm{ADC}}}\right) \cdot \mathrm{TV} \cdot\left(\frac{2 \cdot \mathrm{P}_{\mathrm{ADC}} \cdot \mathrm{R}_{\mathrm{Cap}}}{\mathrm{R}_{\mathrm{Krogh}}^{2}}+\frac{6 \cdot \mathrm{D}_{\mathrm{ADC}}}{\mathrm{R}_{\mathrm{Tumor}}^{2}}\right) \\
& \frac{\mathrm{d}\left(\mathrm{X} 2_{\mathrm{ADC}}\right)}{\mathrm{dt}}=\frac{\mathrm{CLD} D_{\mathrm{ADC}}}{\mathrm{V} 1_{\mathrm{ADC}}} \cdot \mathrm{X} 1_{\mathrm{ADC}}-\frac{\mathrm{CLD} D_{\mathrm{ADC}}}{\mathrm{V} 2_{\mathrm{ADC}}} \cdot \mathrm{X} 2_{\mathrm{ADC}} \\
& \frac{\mathrm{d}\left(\mathrm{C} 1_{\text {Drug }}\right)}{\mathrm{dt}}=-\frac{\mathrm{C} L_{\text {Drug }}}{\mathrm{V} 1_{\text {Drug }}} \cdot \mathrm{C} 1_{\text {Drug }}-\frac{\mathrm{CLD} D_{\text {Drug }}}{\mathrm{V} 1_{\text {Drug }}} \cdot \mathrm{C} 1_{\text {Drug }}+\frac{\mathrm{CLD} D_{\text {Drug }}}{\mathrm{V} 1_{\text {Drug }}} \cdot \mathrm{C} 2_{\text {Drug }} \\
& +\frac{\left(\mathrm{K}_{\mathrm{dec}}^{\mathrm{P}} \cdot \mathrm{X} 1_{\mathrm{ADC}} \cdot \overline{\mathrm{DAR}}\right)}{\mathrm{V} 1_{\text {Drug }}}+\frac{\mathrm{CL} \mathrm{ADC}}{\mathrm{V} \cdot \overline{\mathrm{DAR}} \cdot \frac{\mathrm{X} 1_{\mathrm{ADC}}}{\mathrm{V} 1_{\mathrm{ADC}}}} \\
& -\left(\mathrm{C} 1_{\text {Drug }}-\frac{\text { Drug }_{\mathrm{f}}^{\mathrm{ex}}}{\left(\mathrm{TV} \cdot \varepsilon^{\text {Drug }}\right)}\right) \cdot\left(\frac{2 \cdot \mathrm{P}_{\text {Drug }} \cdot \mathrm{R}_{\text {Cap }}}{\mathrm{R}_{\mathrm{Krogh}}^{2}}+\frac{6 \cdot \mathrm{D}_{\text {Drug }}}{\mathrm{R}_{\text {Tumor }}^{2}}\right) \\
& \frac{\mathrm{d}\left(\mathrm{C} 2_{\text {Drug }}\right)}{\mathrm{dt}}=\frac{\mathrm{CLD} D_{\text {Drug }}}{\mathrm{V} 2_{\text {Drug }}} \cdot \mathrm{C} 1_{\text {Drug }}-\frac{\mathrm{CLD} D_{\text {Drug }}}{\mathrm{V} 2_{\text {Drug }}} \cdot \mathrm{C} 2_{\text {Drug }} \\
& \frac{\mathrm{d}(\overline{\mathrm{DAR}})}{\mathrm{dt}}=-\mathrm{K}_{\mathrm{dec}}^{\mathrm{P}} \cdot \overline{\mathrm{DAR}} \text {. }
\end{aligned}
$$

Above, initial conditions for eqs. 5 and 9 are injected intravenous dose of $\mathrm{ADC}\left(\mathrm{Dose}^{\mathrm{ADC}}\right)$ and initial average DAR of drug formulation $[\overline{\mathrm{DAR}}(0)]$, respectively. Initial conditions for the rest of the equations are zero. Equations associated with the concentration of T-vc-MMAE and amounts of unconjugated MMAE in tumor extracellular space are listed below:

$$
\begin{aligned}
& \frac{\mathrm{d}\left(\mathrm{ADC}_{\mathrm{f}}^{\mathrm{ex}}\right)}{\mathrm{dt}}=\left(\frac{\mathrm{X} 1_{\mathrm{ADC}}}{\mathrm{V} 1_{\mathrm{ADC}}}-\frac{\mathrm{ADC}_{\mathrm{f}}^{\mathrm{ex}}}{\varepsilon^{\mathrm{ADC}}}\right) \cdot\left(\frac{2 \cdot \mathrm{P}_{\mathrm{ADC}} \cdot \mathrm{R}_{\mathrm{Cap}}}{\mathrm{R}_{\mathrm{Krogh}}^{2}}+\frac{6 \cdot \mathrm{D}_{\mathrm{ADC}}}{\mathrm{R}_{\mathrm{Tumor}}^{2}}\right) \\
& +\left(-\mathrm{K}_{\mathrm{on}}^{\mathrm{ADC}} \cdot \frac{\mathrm{ADC}_{\mathrm{f}}^{\mathrm{ex}}}{\varepsilon^{\mathrm{ADC}}} \cdot\left(\mathrm{Ag}_{\mathrm{Ex}}^{\mathrm{N} 87}-\mathrm{ADC}_{\mathrm{b}}^{\mathrm{N} 87}\right)+\mathrm{K}_{\mathrm{off}}^{\mathrm{ADC}} \cdot \mathrm{ADC}_{\mathrm{b}}^{\mathrm{N} 87}\right) \cdot \frac{\mathrm{NC}^{\mathrm{N} 87} \cdot \mathrm{SF}}{\mathrm{TV}} \\
& \quad+\left(-\mathrm{K}_{\mathrm{on}}^{\mathrm{ADC}} \cdot \frac{\mathrm{ADC}_{\mathrm{f}}^{\mathrm{ex}}}{\varepsilon^{\mathrm{ADC}}} \cdot\left(\mathrm{Ag}_{\mathrm{Ex}}^{\mathrm{MCF} 7}-\mathrm{ADC}_{\mathrm{b}}^{\mathrm{MCF7}}\right)+\mathrm{K}_{\mathrm{off}}^{\mathrm{ADC}} \cdot \mathrm{ADC}_{\mathrm{b}}^{\mathrm{MCF} 7}\right) \\
& \frac{\mathrm{NC}^{\mathrm{MCF} 7} \cdot \mathrm{SF}}{\mathrm{TV}}-\mathrm{K}_{\mathrm{dec}}^{\mathrm{p}} \cdot \mathrm{ADC}_{\mathrm{f}}^{\mathrm{ex}}
\end{aligned}
$$$$
\frac{\mathrm{d}\left(\text { Drug }_{\mathrm{f}}^{\text {ex }}\right)}{\mathrm{dt}}=\left(\mathrm{C} 1_{\text {Drug }}-\frac{\text { Drug }_{\mathrm{f}}^{\text {ex }}}{\left(\mathrm{TV} \cdot \varepsilon^{\text {Drug }}\right)}\right) \cdot \mathrm{TV} \cdot\left(\frac{2 \cdot \mathrm{P}_{\text {Drug }} \cdot \mathrm{R}_{\mathrm{Cap}}}{\mathrm{R}_{\mathrm{Krogh}}^{2}}+\frac{6 \cdot \mathrm{D}_{\text {Drug }}}{\mathrm{R}_{\text {Tumor }}^{2}}\right)
$$$$
+\mathrm{K}_{\mathrm{dec}}^{\mathrm{p}} \cdot \mathrm{ADC}_{\mathrm{f}}^{\mathrm{ex}} \cdot \overline{\mathrm{DAR}} \cdot \mathrm{TV}+\left(\mathrm{K}_{\mathrm{dec}}^{\mathrm{p}} \cdot \mathrm{ADC}_{\mathrm{b}}^{\mathrm{N} 87} \cdot \overline{\mathrm{DAR}}+\mathrm{K}_{\mathrm{out}}^{\mathrm{Drug}} \cdot \operatorname{Drug}_{\mathrm{f}}^{\mathrm{N} 87}\right)
$$$$
\cdot \mathrm{NC}^{\mathrm{N} 87} \cdot \mathrm{SF}+\left(\mathrm{K}_{\mathrm{dec}}^{\mathrm{p}} \cdot \mathrm{ADC}_{\mathrm{b}}^{\mathrm{MCF} 7} \cdot \overline{\mathrm{DAR}}+\mathrm{K}_{\mathrm{out}}^{\mathrm{Drug}} \cdot \operatorname{Drug}_{\mathrm{f}}^{\mathrm{MCF} 7}\right)
$$$$
\cdot \mathrm{NC}^{\mathrm{MCF} 7} \cdot \mathrm{SF}-\mathrm{K}_{\mathrm{in}}^{\mathrm{Drug}} \cdot \mathrm{NC}^{\mathrm{N} 87} \cdot\left(\frac{\mathrm{V}^{\mathrm{N} 87}}{\mathrm{TV} \cdot \varepsilon^{\text {Drug }}}\right) \cdot \operatorname{Drug}_{\mathrm{f}}^{\mathrm{ex}}-\mathrm{K}_{\mathrm{in}}^{\text {Drug }}
$$$$
\cdot \mathrm{NC}^{\mathrm{MCF} 7} \cdot\left(\frac{\mathrm{V}^{M C F 7}}{\mathrm{TV} \cdot \varepsilon^{\mathrm{Drug}}}\right) \cdot \operatorname{Drug}_{\mathrm{f}}^{\mathrm{ex}} \text {. }
$$

Equations associated with cellular disposition of T-vc-MMAE and unconjugated MMAE in each cell type " $i$ " (where " $i$ " can be either N87 or GFP-MCF7 cell) are provided below. Concentrations are represented in the form of number of molecules of each analyte per single tumor cell:

$$
\begin{aligned}
\frac{\mathrm{d}\left(\mathrm{ADC}_{\mathrm{b}}^{\mathrm{i}}\right)}{\mathrm{dt}}= & \mathrm{K}_{\mathrm{on}}^{\mathrm{ADC}} \cdot \frac{\mathrm{ADC}_{\mathrm{f}}^{\mathrm{ex}}}{\varepsilon^{\mathrm{ADC}}} \cdot\left(A g_{E x}^{i}-\mathrm{ADC}_{\mathrm{b}}^{\mathrm{i}}\right)-\mathrm{K}_{\mathrm{off}}^{\mathrm{ADC}} \cdot \mathrm{ADC}_{\mathrm{b}}^{\mathrm{i}}- \\
& \left(\mathrm{K}_{\mathrm{dec}}^{\mathrm{p}}+\mathrm{K}_{\mathrm{int}}^{\mathrm{ADC}}\right) \cdot \mathrm{ADC}_{\mathrm{b}}^{\mathrm{i}}-\frac{\mathrm{Ln} 2}{\mathrm{DT}^{\mathrm{i}}} \cdot \mathrm{ADC}_{\mathrm{b}}^{\mathrm{i}}
\end{aligned}
$$

$$
\begin{aligned}
& \frac{\mathrm{d}\left(\mathrm{ADC}_{\text {lyso }}^{\mathrm{i}}\right)}{\mathrm{dt}}=\mathrm{K}_{\mathrm{int}}^{\mathrm{ADC}} \cdot \mathrm{ADC}_{\mathrm{b}}^{\mathrm{i}}-\mathrm{K}_{\mathrm{deg}}^{\mathrm{ADC}} \cdot \mathrm{ADC}_{\text {lyso }}^{\mathrm{i}}-\frac{\mathrm{Ln} 2}{\mathrm{DT}^{\mathrm{i}}} \cdot \mathrm{ADC}_{\text {lys }}^{\mathrm{i}} \\
& \frac{\mathrm{d}\left(\operatorname{Drug}_{\mathrm{f}}^{\mathrm{i}}\right)}{\mathrm{dt}}=\mathrm{K}_{\mathrm{deg}}^{\mathrm{ADC}} \cdot \mathrm{ADC}_{\text {lyso }}^{\mathrm{i}} \cdot \overline{\mathrm{DAR}}-\mathrm{K}_{\text {out }}^{\mathrm{Drug}} \cdot \operatorname{Drug}_{\mathrm{f}}^{\mathrm{i}}-\mathrm{K}_{\mathrm{on}}^{\mathrm{Tub}} \cdot \operatorname{Drug}_{\mathrm{f}}^{\mathrm{i}} \\
& \cdot\left(\operatorname{Tub}_{\text {tot }}^{\mathrm{i}}-\operatorname{Drug}_{\mathrm{b}}^{\mathrm{i}}\right)+\mathrm{K}_{\mathrm{off}}^{\mathrm{Tub}} \cdot \operatorname{Drug}_{\mathrm{b}}^{\mathrm{i}}+\mathrm{K}_{\mathrm{in}}^{\mathrm{Drug}} \cdot\left(\frac{\mathrm{V}^{\mathrm{i}}}{\mathrm{TV} \cdot \varepsilon^{\text {Drug }}}\right) \cdot\left(\frac{\operatorname{Drug}_{\mathrm{f}}^{\mathrm{ex}}}{\mathrm{SF}}\right) \\
& -\frac{\mathrm{Ln} 2}{\mathrm{DT}^{\mathrm{i}}} \cdot \operatorname{Drug}_{\mathrm{f}}^{\mathrm{i}} \\
& \begin{aligned}
\frac{d\left(\operatorname{Drug}_{b}^{i}\right)}{d t} & =K_{o n}^{\text {Tub }} \cdot \operatorname{Drug}_{f}^{i} \cdot\left(\operatorname{Tub}_{\text {tot }}^{\mathrm{i}}-\operatorname{Drug}_{b}^{\mathrm{i}}\right)-\mathrm{K}_{\text {off }}^{\mathrm{Tub}} \cdot \operatorname{Drug}_{\mathrm{b}}^{\mathrm{i}} \\
& -\frac{\mathrm{Ln}^{2}}{\mathrm{DT}^{\mathrm{i}}} \cdot \text { Drugg }_{\mathrm{b}}^{\mathrm{i}} .
\end{aligned}
\end{aligned}
$$

Above, the initial conditions for eqs. 10-15 are zero.

Calculation of Intracellular Tubulin Occupancy in Each Cell Type. Upon integration of the tumor distribution model with the single-cell PK model, the percent occupancy of tubulin by MMAE inside each tumor cell can be calculated by dividing the number of tubulin-bound MMAE molecules with the number of total tubulin molecules inside each cell type " $i$ " (where " $i$ " can be either N87 or GFPMCF7 cell). The relevant equation is provided below:

$$
\operatorname{Occ}_{\text {tub }}^{\mathrm{i}}=\left(\frac{\operatorname{Drug}_{\mathrm{b}}^{\mathrm{i}}}{\operatorname{Tub}_{\text {tot }}^{\mathrm{i}}}\right) \cdot 100 .
$$


Driving Direct Killing and Bystander Effect of ADC Using Intracellular Tubulin Occupancy. TGI data generated using different dose levels of ADC in all three mouse models (i.e., N87, GFP-MCF7, and coculture) were used to develop a systems PK-PD model that could account for direct killing and bystander effect of ADCs in vivo (Fig. 5). First, the coculture tumor PK model was used to predict intracellular occupancy of tubulin in each cell type. The intracellular occupancy was then used to drive the cytotoxicity of each cell type, using a nonlinear killing function that shuttles the growing tumor cells into nongrowing phases, which eventually die. This model is also known as the "celldistribution model" (Yang et al., 2010). It was assumed that among all different cell populations (growing or nongrowing), the processing of $\mathrm{ADC}$ was active, and upon the death of each cell type, the intracellular content (either intact T-vc-MMAE or free MMAE) comes out and becomes the part of extracellular space. The released intracellular content could then redistribute back into other cells or diffuse out into the systemic circulation via surface or vascular exchange processes (Fig. 5). The resulting equations characterizing tumor growth dynamics of each cell type " $i$ " (where " $i$ " can be either N87 or GFP-MCF7 cell) are provided below:

Growth rate:

$$
\mathrm{Kg}^{i}=\left(\frac{\mathrm{Ln} 2}{\mathrm{DT}^{\mathrm{i}}}\right)
$$

Killing rate:

$$
\begin{gathered}
\mathrm{Kill}^{\mathrm{i}}=\left\{\frac{\mathrm{Kmax} \cdot\left(\mathrm{Occ}_{\text {tub }}^{\mathrm{i}}\right)^{\gamma}}{(\mathrm{KC} 50)^{\gamma}+\left(\mathrm{Occ}_{\mathrm{tub}}^{\mathrm{i}}\right)^{\gamma}}\right\} \\
\frac{\mathrm{d}\left(\mathrm{TV}_{\mathrm{i}}^{1}\right)}{\mathrm{dt}}=\left(\mathrm{Kg}^{\mathrm{i}}-\mathrm{Kill}^{\mathrm{i}}\right) \cdot \mathrm{TV}_{\mathrm{i}}^{1} \\
\frac{\mathrm{d}\left(\mathrm{TV}_{\mathrm{i}}^{2}\right)}{\mathrm{dt}}=\mathrm{Kill}^{\mathrm{i}} \cdot \mathrm{TV}_{\mathrm{i}}^{1}-\frac{1}{\tau} \cdot \mathrm{TV}_{\mathrm{i}}^{2} \\
\frac{\mathrm{d}\left(\mathrm{TV}_{\mathrm{i}}^{3}\right)}{\mathrm{dt}}=\frac{1}{\tau} \cdot\left(\mathrm{TV}_{\mathrm{i}}^{2}-\mathrm{TV}_{\mathrm{i}}^{3}\right) \\
\frac{\mathrm{d}\left(\mathrm{TV}_{\mathrm{i}}^{4}\right)}{\mathrm{dt}}=\frac{1}{\tau} \cdot\left(\mathrm{TV}_{\mathrm{i}}^{3}-\mathrm{TV}_{\mathrm{i}}^{4}\right) \\
\mathrm{TV}_{\mathrm{mm} 3}^{\mathrm{i}}=\mathrm{TV}_{\mathrm{i}}^{1}+\mathrm{TV}_{\mathrm{i}}^{2}+\mathrm{TV}_{\mathrm{i}}^{3}+\mathrm{TV}_{\mathrm{i}}^{4} \\
\mathrm{TV}_{\mathrm{mm} 3}=\mathrm{TV}_{\mathrm{mm} 3}^{\mathrm{N} 87}+\mathrm{TV}_{\mathrm{mm} 3}^{\mathrm{MCF} 7} .
\end{gathered}
$$

The equations for concentration of T-vc-MMAE (eq. 10) and amount of MMAE (eq. 11) in the tumor extracellular space were updated to have inputs from intracellular content of the last transit compartment $\left(\mathrm{TV}_{\mathrm{i}}^{4}\right)$ for each cell-type (N87 or GFP-MCF7). The resulting equations are provided below:

$$
\begin{aligned}
& \frac{\mathrm{d}\left(\mathrm{ADC}_{\mathrm{f}}^{\mathrm{ex}}\right)}{\mathrm{dt}}=\left(\frac{\mathrm{X} 1_{\mathrm{ADC}}}{\mathrm{V} 1_{\mathrm{ADC}}}-\frac{\mathrm{ADC}_{\mathrm{f}}^{\mathrm{ex}}}{\varepsilon^{\mathrm{ADC}}}\right) \cdot\left(\frac{2 \cdot \mathrm{P}_{\mathrm{ADC}} \cdot \mathrm{R}_{\mathrm{Cap}}}{\mathrm{R}_{\mathrm{Krogh}}^{2}}+\frac{6 \cdot \mathrm{D}_{\mathrm{ADC}}}{\mathrm{R}_{\text {Tumor }}^{2}}\right) \\
& +\left(-\mathrm{K}_{\mathrm{on}}^{\mathrm{ADC}} \cdot \frac{\mathrm{ADC}_{\mathrm{f}}^{\mathrm{ex}}}{\varepsilon^{\mathrm{ADC}}} \cdot\left(\mathrm{Ag}_{\mathrm{Ex}}^{\mathrm{N} 87}-\mathrm{ADC}_{\mathrm{b}}^{\mathrm{N} 87}\right)+\mathrm{K}_{\mathrm{off}}^{\mathrm{ADC}} \cdot \mathrm{ADC}_{\mathrm{b}}^{\mathrm{N} 87}\right) \\
& \frac{\mathrm{NC}^{\mathrm{N} 87} \cdot \mathrm{SF}}{\mathrm{TV}} \\
& +\left(-\mathrm{K}_{\mathrm{on}}^{\mathrm{ADC}} \cdot \frac{\mathrm{ADC}_{\mathrm{f}}^{\mathrm{ex}}}{\varepsilon^{\mathrm{ADC}}} \cdot\left(\mathrm{Ag}_{\mathrm{Ex}}^{\mathrm{MCF} 7}-\mathrm{ADC}_{\mathrm{b}}^{\mathrm{MCF} 7}\right)+\mathrm{K}_{\mathrm{off}}^{\mathrm{ADC}} \cdot \mathrm{ADC}_{\mathrm{b}}^{\mathrm{MCF} 7}\right) \\
& \cdot \frac{\mathrm{NC}^{\mathrm{MCF} 7} \cdot \mathrm{SF}}{\mathrm{TV}}-\mathrm{K}_{\mathrm{dec}}^{\mathrm{p}} \cdot \mathrm{ADC}_{\mathrm{f}}^{\mathrm{ex}}+\frac{1}{\tau} \cdot \mathrm{TV}_{\mathrm{N} 87}^{4} \cdot 10^{5} \cdot\left(\mathrm{ADC}_{\mathrm{b}}^{\mathrm{N} 87}+\mathrm{ADC}_{\mathrm{lyso}}^{\mathrm{N} 87}\right) \\
& \cdot \frac{\mathrm{SF}}{\mathrm{TV}}+\frac{1}{\tau} \cdot \mathrm{TV}_{\mathrm{MCF} 7}^{4} \cdot 10^{5} \cdot\left(\mathrm{ADC}_{\mathrm{b}}^{\mathrm{MCF} 7}+\mathrm{ADC}_{\text {lyso }}^{\mathrm{MCF7}}\right) \cdot \frac{\mathrm{SF}}{\mathrm{TV}}
\end{aligned}
$$

$$
\begin{aligned}
& \frac{\mathrm{d}\left(\mathrm{Drug}_{\mathrm{f}}^{\mathrm{ex}}\right)}{\mathrm{dt}}=\left(\mathrm{C} 1_{\mathrm{Drug}}-\frac{\mathrm{Drug}_{\mathrm{f}}^{\mathrm{ex}}}{\mathrm{TV} \cdot \varepsilon^{\mathrm{Drug}}}\right) \cdot \mathrm{TV} \cdot\left(\frac{2 \cdot \mathrm{P}_{\text {Drug }} \cdot \mathrm{R}_{\mathrm{Cap}}}{\mathrm{R}_{\mathrm{Krogh}}^{2}}+\frac{6 \cdot \mathrm{D}_{\text {Drug }}}{\mathrm{R}_{\text {Tumor }}^{2}}\right) \\
& +\mathrm{K}_{\mathrm{dec}}^{\mathrm{p}} \\
& \cdot \mathrm{ADV}_{\mathrm{f}}^{\mathrm{ex}} \cdot \overline{\mathrm{DAR}} \cdot \mathrm{TV}+\left(\mathrm{K}_{\mathrm{dec}}^{\mathrm{p}} \cdot \mathrm{ADV}_{\mathrm{b}}^{\mathrm{N} 87} \cdot \overline{\mathrm{DAR}}+\mathrm{K}_{\mathrm{out}}^{\mathrm{Drug}} \cdot \mathrm{Drug}_{\mathrm{f}}^{\mathrm{N} 87}\right) \cdot \mathrm{NC}^{\mathrm{N} 87} \\
& \cdot \mathrm{SF}+\left(\mathrm{K}_{\mathrm{dec}}^{\mathrm{p}} \cdot \mathrm{ADC}_{\mathrm{b}}^{\mathrm{MCF} 7} \cdot \overline{\mathrm{DAR}}+\mathrm{K}_{\mathrm{out}}^{\mathrm{Drug}} \cdot \mathrm{Drug}_{\mathrm{f}}^{\mathrm{MCF} 7}\right) \cdot \mathrm{NC}^{\mathrm{MCF} 7} \cdot \mathrm{SF} \\
& -\mathrm{K}_{\mathrm{in}}^{\mathrm{Drug}} \cdot \mathrm{NC}^{\mathrm{N} 87} \cdot\left(\frac{\mathrm{V}^{\mathrm{N} 87}}{\mathrm{TV} \cdot \varepsilon^{\mathrm{Drug}}}\right) \cdot \mathrm{Drug}_{\mathrm{f}}^{\mathrm{ex}}-\mathrm{K}_{\mathrm{in}}^{\mathrm{Drug}} \cdot \mathrm{NC}^{\mathrm{MCF} 7} \cdot\left(\frac{\mathrm{V}^{M C F 7}}{\mathrm{TV}^{\mathrm{Drug}}}\right) \\
& \cdot \mathrm{Drug}_{\mathrm{f}}^{\mathrm{ex}}+\frac{1}{\tau} \cdot \mathrm{TV}_{\mathrm{N} 87}^{4} \cdot 10^{5} \cdot\left(\mathrm{Drug}_{\mathrm{f}}^{\mathrm{ex}}+\mathrm{Drug}_{\mathrm{b}}^{\mathrm{ex}}\right) \cdot \mathrm{SF}+\frac{1}{\tau} \cdot \mathrm{TV}_{\mathrm{MCF} 7}^{4} \\
& \cdot 10^{5} \cdot\left(\mathrm{Drug}_{\mathrm{f}}^{\mathrm{MCF} 7}+\mathrm{Drug}_{\mathrm{b}}^{\mathrm{MCF} 7}\right) \cdot \mathrm{SF}
\end{aligned}
$$

\section{Parameter Estimation, Model Fitting, and Simulations}

A Priori Prediction of Tumor Pharmacokinetics of T-vc-MMAE in the Coculture Tumor Model. Our previously developed tumor PK model for N87 and GFP-MCF7 xenografts (Singh et al., 2019) was evolved to account for heterogeneous tumor cell population (Fig. 3). Two distinct populations of tumor cell types were assumed, and previously published parameters (Table 1) were used to a priori predict tumor exposure of different ADC analytes (i.e., free MMAE, total MMAE, and total Trastuzumab). The PK was predicted following intravenous administration of $10 \mathrm{mg} / \mathrm{kg}$ T-vc-MMAE in a mouse bearing 50:50 coculture tumor.

Simulations of Free and Total MMAE Exposures at Different Dose Levels in Three Mouse Models. After validation of the tumor PK model for the heterogeneous tumor, simulations were performed to predict free and total MMAE exposures in the heterogeneous tumor after intravenous dose of $0.1,1$, and $10 \mathrm{mg} / \mathrm{kg}$ dose. The predicted PK profiles in 50:50 coculture tumor were compared with the PK profiles in N87 and GFP-MCF7 monoculture tumors.

Simulations for Intracellular Tubulin Occupancy within Each Cell Type in a Heterogenous Tumor. Simulations for percent tubulin occupancy in each cell type (i.e., N87 and GFP-MCF7) of 50:50 coculture tumor were performed at $0.1-10 \mathrm{mg} / \mathrm{kg}$ doses of T-vcMMAE. In addition, an improvement in the percentage tubulin occupancy of GFP-MCF7 (HER2-low) cell with increasing fractions of N87 (HER2-high) cells in a heterogeneous tumor was also simulated at $0.1-10 \mathrm{mg} / \mathrm{kg}$ doses. The improvement in percentage tubulin occupancy of $\mathrm{Ag}$ - cells is used as a driver for $\mathrm{ADC}$ bystander effect.

Simultaneous Fittings of TGI Data Sets from N87, GFP-MCF, and 50:50 Coculture Xenografts.

Step 1: Individual tumor growth profiles of N87 and GFP-MCF7 xenografts from the control group were fitted to an exponential growth model (eq. 17). Because of the observed variability in the individual tumor growth profiles, a mean tumor doubling time $\left(\mathrm{DT}^{\mathrm{N} 87}, \mathrm{DT}^{\mathrm{MCF} 7}\right)$ and intersubject variability with log-normal distribution ( $\mathrm{IIV}^{\mathrm{DT}^{\mathrm{N} 87}}, \mathrm{IIV}^{\mathrm{DT}}{ }^{\mathrm{MCF} 7}$ ) were estimated for each xenograft (N87 and GFP-MCF7). To characterize the tumor growth of the coculture xenografts, it was assumed that at time 0 , the composition of the heterogeneous tumor included 50:50 ratio of both the cell types. The estimated mean doubling times associated with N87 and GFP-MCF7 tumors were fixed to previously obtained values, and the individual tumor growth profiles in the control group for all three mouse models (N87, GFP-MCF7, and coculture) were simultaneously fitted to precisely estimate the parameters associated with intersubject variability in the growth of N87 and GFP-MCF7 cells. 
TABLE 1

A list of literature-derived or model-estimated parameters used for the systems PK-PD model of T-vc-MMAE

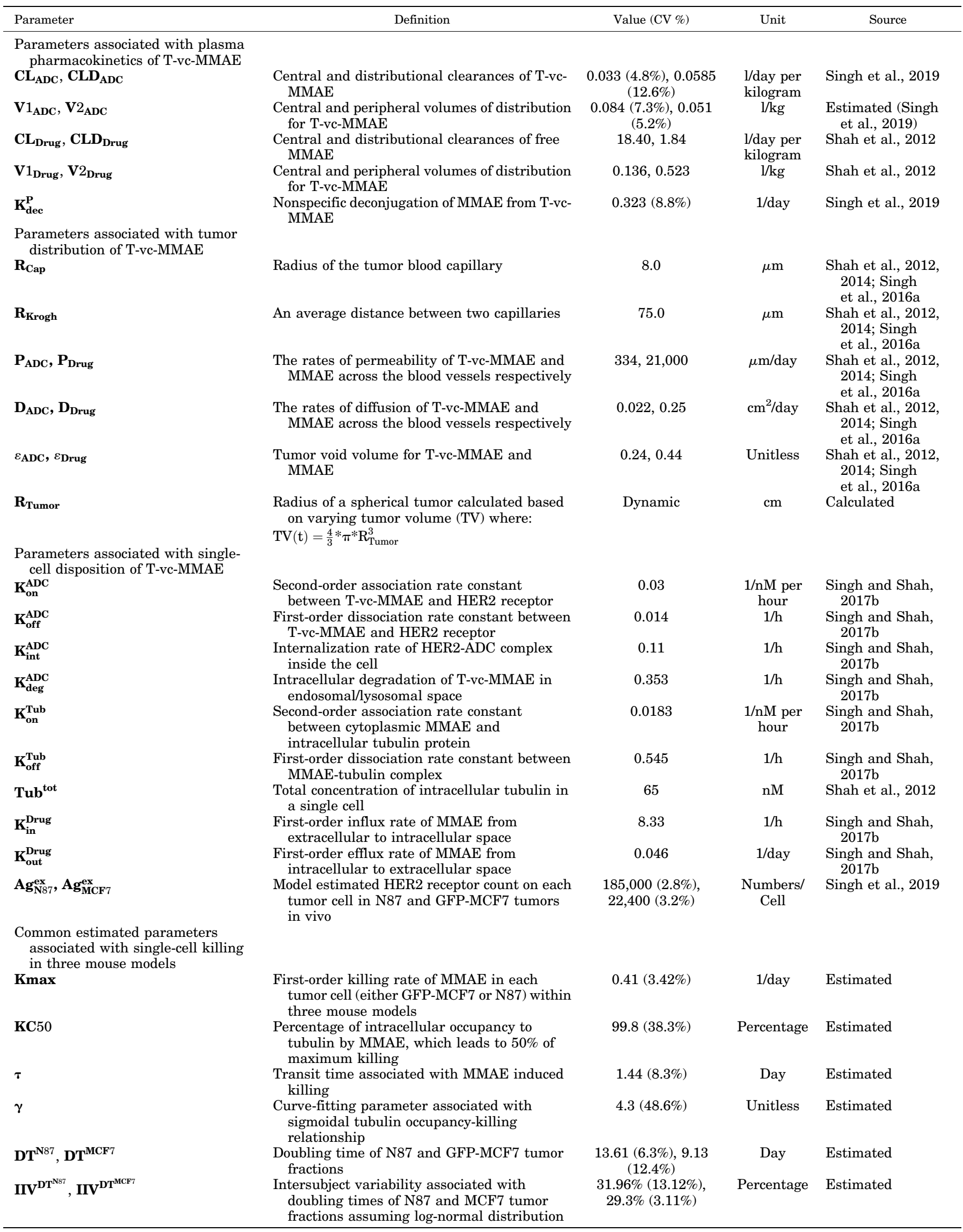


Step 2: The final systems PK-PD model for T-vc-MMAE (Fig. 5) was used to simultaneously characterize all the TGI profiles from all three tumor models. It was assumed that all the variability associated with TGI profiles was stemming from the variable in the growth. Consequently, tumor doubling times and associated intersubject variability were fixed, and only the common efficacy parameters (Kmax, KC50, $\tau$ ) pertaining to MMAE-induced tumor cell killing were estimated.

Simulations for Tumor Growth Profiles of N87 and GFP-MCF7 Fractions in a Heterogeneous Model. Once the full tumor PK-PD model (Fig. 5) was validated by characterizing the entire TGI data set, simulations were performed to comparatively evaluate growth profiles of N87 and GFP-MCF7 fractions (within a heterogeneous tumor) in comparison with whole 50:50 coculture tumor after administration of 1 and $10 \mathrm{mg} / \mathrm{kg}$ i.v. T-vc-MMAE. For these set of simulations, common estimated efficacy parameters and the mean doubling time parameters associated with N87 and GFP-MCF7 growth were used without consideration of any intersubject variability.

Simulations for Model-Predicted "Bystander Effect" Following Single or Fractionated Dosing Regimen. Because the main mechanism of bystander effect is enhanced killing of antigen-low (GFP-MCF7) cells in the presence of antigen-high (N87) cells, this effect was investigated by performing simulations using the final PKPD model. Tumor growth profiles for "MCF7 fraction" of a heterogeneous tumor were simulated at $1-10 \mathrm{mg} / \mathrm{kg}$ doses, which were either administered as a single dose or in a fractionated dosing regimen (four equal doses administered every 4 days, Q4dX4). The improvement in the efficacy against "MCF7 fraction" of a heterogeneous tumor was analyzed to identify the dose or dosing regimen that leads to the maximum bystander effect. These sets of simulations were performed without any intersubject variability.
Effect of Different Fractionated Dosing Regimens on Maximizing TGI of 50:50 Coculture Xenograft. A clinically relevant dose of $3.6 \mathrm{mg} / \mathrm{kg}$ was selected, and its effect was evaluated on the TGI of a heterogeneous tumor with 50:50 composition of $\mathrm{N} 87$ and GFP-MCF7 cells. The selected dose $(3.6 \mathrm{mg} / \mathrm{kg})$ was either administered as a single intravenous dose or as a fractionated dose in the form of $1.2 \mathrm{mg} / \mathrm{kg}$ given $\mathrm{Q} 4 \mathrm{dX} 3$ or $0.6 \mathrm{mg} / \mathrm{kg}$ given $\mathrm{Q} 4 \mathrm{dX} 6$. Tumor growth profiles for the "whole" heterogeneous tumor and its N87 and GFPMCF7 fractions were simulated and compared with the control profiles.

Data Analysis. Initial model development and simulation work was performed using Berkeley Madonna (University of California at Berkeley, CA). Model fitting to TGI profiles was accomplished using Stochastic Approximation Expectation Maximization algorithm in Monolix version 8 (Lixoft) (Lavielle and Mentre, 2007).

\section{Results}

\section{IHC Analysis of the Heterogeneous Tumor}

Figure 1A shows a representative fluorescent image of the histologic section of heterogeneous tumor. The experiment was done in triplicate, and Supplemental Fig. 1 shows the images of all three coculture tumors. Fluorescent images of DAPI (in blue) and GFP (in green) were merged to identify the regions of interest. It was observed that the intensity of GFP signal was highly variable and heterogeneous. There were regions of densely populated GFP-MCF7 cells (Fig. 1A1) and regions where DAPI signal was predominant (Fig. 1A2), suggesting the presence of N87 cells. It was also observed that the two cell types were not "well mixed" and grew in clusters. Figure 1B shows the results of

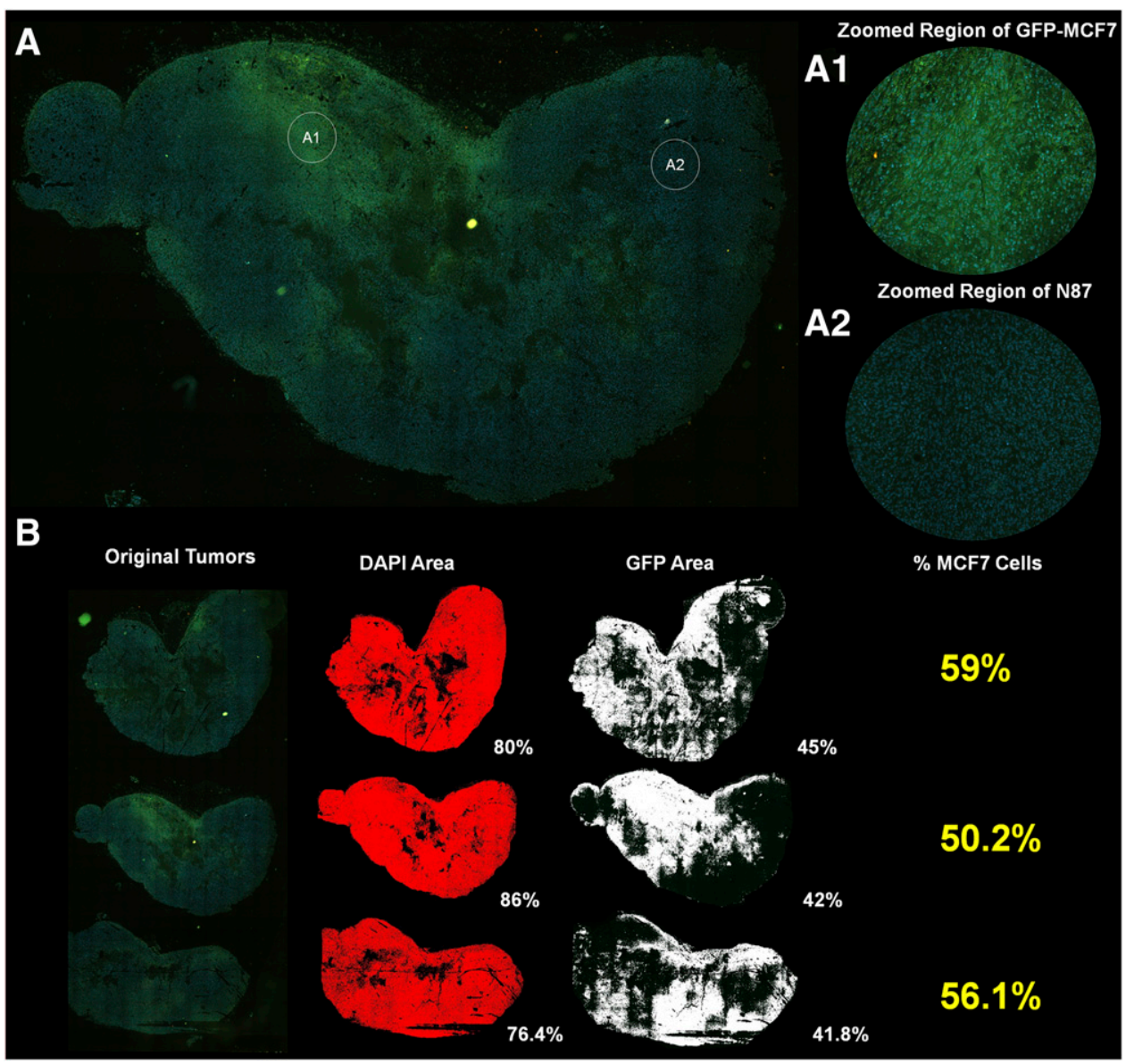

Fig. 1. Imaging analysis of coculture tumors grown using a mixture of N87 and GFP-MCF7 cells and harvested at a volume of $\sim 350-400 \mathrm{~mm}^{3}$. (A) A representative image of the middle section of the tumor. The green signal represents GFP-transfected MCF7 cells, and the blue signal from DAPI represent nucleus of all tumor cells. (A1) A zoomed region of the tumor populated with GFP-MCF7 cells, which have both "green" and "blue" signals. (A2) A zoomed region of the tumor populated with N87 cells, which have only "blue" signal. (B) A quantitative analysis of the middle sections of three tumors to calculate the percentage of GFPMCF7 cells within a heterogeneous tumor. 

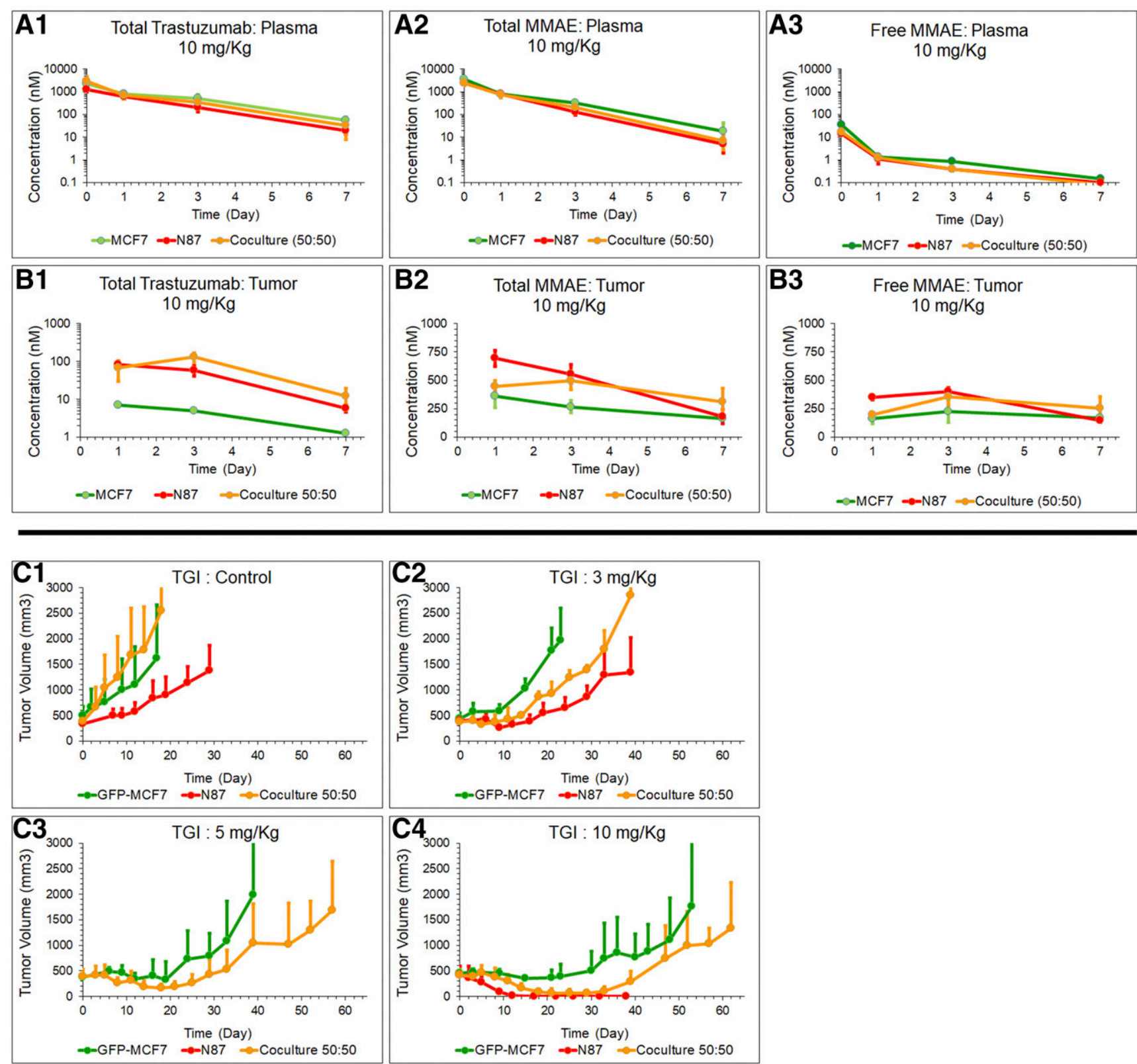

Fig. 2. (A) Plasma PK of all three ADC analytes in GFP-MCF7, N87, and coculture tumor-bearing mice after $10 \mathrm{mg} / \mathrm{kg}$ i.v. dose of T-vc-MMAE. (A1) Total Trastuzumab, (A2) total MMAE, and (A3) free MMAE. (B) Tumor PK of all three ADC analytes in GFP-MCF7, N87, and coculture tumor-bearing mice after $10 \mathrm{mg} / \mathrm{kg}$ i.v. dose of T-vc-MMAE. (B1) total trastuzumab, (B2) total MMAE, and (B3) free MMAE. (C) Tumor growth profiles for all three xenograft models. (C1) control, (C2) $3 \mathrm{mg} / \mathrm{kg}$ T-vc-MMAE, (C3) $5 \mathrm{mg} / \mathrm{kg}$ T-vc-MMAE, and (C4) $10 \mathrm{mg} / \mathrm{kg}$ T-vc-MMAE.

semiquantitative analysis of the sections by ImageJ software. The fluorescent images from DAPI and GFP were isolated, and percentage of the tumor area emitting each fluorescence signal was determined. It was observed that $\sim 80 \%$ of the total tumor area within the section was covered with tumor cells. In addition, it was observed that $\sim 43 \%$ of the tumor area was covered with GFP-labeled MCF7 cells. As such, it was calculated that $~ 53 \%$ of the tumor cells were GFP-MCF7 cells. Based on these estimates, a 50:50 composition of two cell types was assumed in the coculture tumors for the modeling work.

\section{Plasma and Tumor Pharmacokinetics of T-vc-MMAE}

Figure 2A depicts plasma PK profiles of total trastuzumab (Fig. 2A1), total MMAE (Fig. 2A2), and free MMAE (Fig. 2A3) in all three tumor models using validated bioanalytical techniques (Supplemental Figs. 16 and 17; Supplemental Tables 1-4). As shown in the figure, there was no notable difference between the plasma PK profiles in different tumor models, suggesting the presence of tumor does not significantly contribute toward systemic PK of ADC. Noncompartmental analysis of the plasma PK profile of T-vc-MMAE in SCID mice has been reported by us earlier (Singh et al., 2019). Figure 2B depicts tumor PK of T-vc-MMAE in all three mouse models. It was observed that the tumor exposures of total trastuzumab (Fig. 2B1), total MMAE (Fig. 2B2), and free MMAE (Fig. 2B3) were higher in HER2-high N87 xenografts compared with HER2-low GFP-MCF7 xenografts. Within the coculture tumor, it was observed that total Trastuzumab profile was very similar to HER2-high N87 tumors. However, 
free MMAE and total MMAE profiles initially started at the same exposure levels as GFP-MCF7 tumors and then gradually reached to a level similar to N87 tumors, with a prolonged Tmax (time taken to achieve maximum exposure, Cmax) in comparison with both $\mathrm{N} 87$ and GFP-MCF7 tumors. Interestingly, at the last time point (168 hours) the exposure of free MMAE and total MMAE in 50:50 coculture tumors was higher than both N87 and GFP-MCF7 tumors. This may stem from the processing of ADCs in Ag-high cells, followed by the release of free drug in the extracellular space, which could then accumulate in the intact Ag-low cells.

\section{TGI Studies}

Figure 2C shows mean tumor growth profiles for N87, GFPMCF7, and 50:50 coculture tumors. It was observed that although there was a considerable variability in the growth of individual tumors, in general, N87 control tumors grew slower compared with GFP-MCF7 and 50:50 coculture control tumors. Because GFP-MCF7 cells are fast growing, one would expect that they would take over the 50:50 coculture tumor over time. Consequently, the growth of 50:50 coculture tumors was similar (Fig. 2C1) to GFP-MCF7 tumors. Within the 3$\mathrm{mg} / \mathrm{kg}$ treatment group, it was observed that initially the growth inhibition for 50:50 coculture tumor was similar to N87 tumors, and once the killing signal diminished, the coculture tumors grew at a faster rate similar to GFP-MCF7 tumors. In the $5-\mathrm{mg} / \mathrm{kg}$ group, as well, coculture tumors regressed faster than GFP-MCF7 tumors, but once the killing signal was diminished, they grew similar to GFPMCF7 tumors. In the 10-mg/kg group (Fig. 2C4), HER2-high N87 tumors declined much faster than coculture tumors, which in the beginning showed a profile similar to GFP-MCF7 tumors. However, the coculture tumors eventually showed a delayed decline at a level similar to N87 tumors before growing back up at a rate similar to GFP-MCF7 tumors. This observation matches the tumor PK data generated at $10 \mathrm{mg} / \mathrm{kg}$ dose, for which it was observed that MMAE exposure in the coculture tumors starts at the level like GFP-MCF7 tumors, but once the $\mathrm{ADC}$ is processed by $\mathrm{N} 87$ cells, the exposure level goes up to N87 tumor levels. These data also accentuate the fact that a sustained presence of high antigen-expressing cells is required to achieve prolonged bystander effect by $\mathrm{ADCs}$.
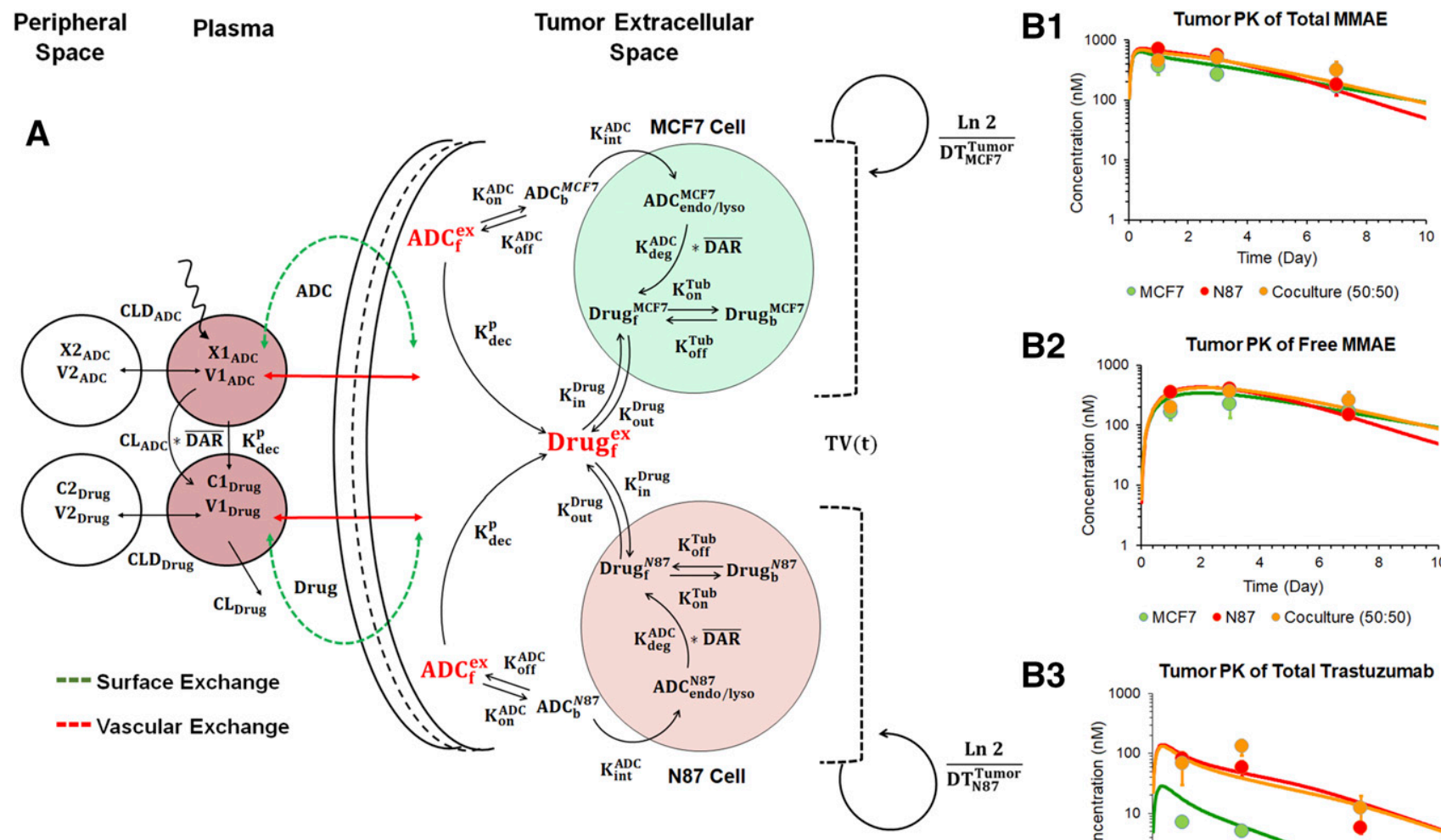

32
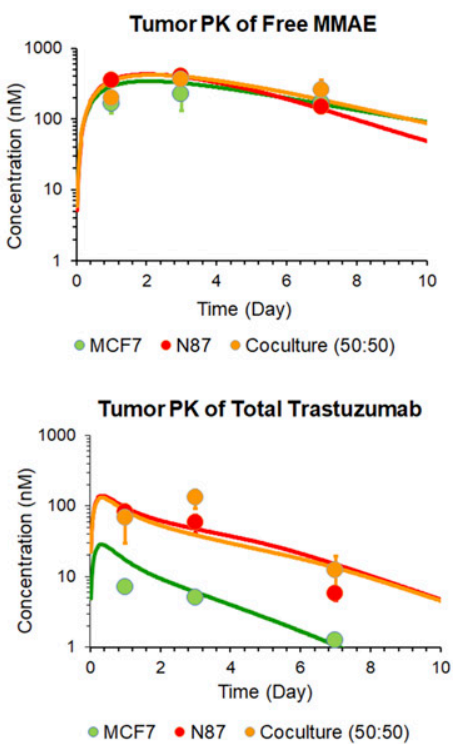

Fig. 3. (A) A schematic diagram of a systems pharmacokinetic model developed to characterize T-vc-MMAE PK in a heterogeneous tumor containing N87 and GFP-MCF7 cells. For plasma PK, disposition of T-vc-MMAE in systemic and peripheral spaces is characterized using a two-compartment model with linear clearance from the central compartment. Processes associated with nonspecific deconjugation of MMAE and catabolic clearance of T-vcMMAE contribute to the formation of free MMAE, which is also characterized using a two-compartment model with distribution to peripheral tissues and linear clearance from the central compartment. For tumor distribution, distribution of T-vc-MMAE and free MMAE from the central compartment to tumor extracellular space was assumed to be driven by two diffusive processes: surface and vascular exchange. Single cell disposition in two cell types: Once in the extracellular space, T-vc-MMAE was assumed to distribute to both N87 and GFP-MCF7 cells, which have distinct growth rates. Once bound to HER2 receptors on the cell, T-vc-MMAE can internalize into endosomal/lysosomal space of each cell. Upon enzymatic degradation and linker cleavage, free MMAE is released in the cytoplasmic space, which can either bind to intracellular tubulin or freely exchange within the extracellular space using influx and efflux rates. Once in tumor extracellular space, free MMAE can either distribute to other cells or diffuse out into the systemic circulation. (B) A priori predictions made by the tumor PK model for all three ADC analytes in the three tumor models. (B1) total MMAE, (B2) free MMAE, and (B3) total trastuzumab. 
Development of the Systems PK-PD Model to Characterize In Vivo Bystander Effect of ADC

Plasma Pharmacokinetic Model for T-vc-MMAE. Plasma PK profiles of all three analytes from all three mouse models was fitted simultaneously using our previously published integrated plasma PK model for ADCs. The model fitted profiles (Supplemental Fig. 3) and relevant parameter values and estimates are provided in Table 1.

A Priori Prediction of Tumor Pharmacokinetics of T-vc-MMAE in a Heterogeneous Tumor. In the past, we have a combined single-cell disposition model of $\mathrm{ADC}$ with a mechanistic tumor distribution model to characterize the exposure of different T-vc-MMAE analytes in GFP-MCF7 and N87 tumors (Singh and Shah, 2017b). All the parameters of this model were previously known or obtained from in vitro modeling (Singh and Shah, 2017b), and only the parameters related to the accessibility of HER2 receptors within the tumor were estimated. In the past, we have also presented the global sensitivity analysis of this model. Here, we have expanded this model to account for tumor heterogeneity. All the parameters associated with plasma $\mathrm{PK}$, tumor distribution, and cellular disposition of T-vc-MMAE were a priori known. Based on the semiquantitative IHC analysis, composition of two cell types within the heterogeneous tumor was assumed to be 50:50. Using the model shown in Fig. 3A, equations described in the Materials and Methods section, and known model parameters shown in Table 1, tumor exposure of the three T-vcMMAE analytes in the heterogeneous tumor was a priori predicted. Figure 3B shows the predicted PK profiles superimposed over the observed data. It was found that the coculture PK model was able to a priori predict the exposure of different $\mathrm{ADC}$ analytes in the heterogeneous tumor reasonably well.

Simulation of Free and Total MMAE Exposure at Different Dose Levels in the Three Tumor Models. The final model was used to simulate the effect of different ADC doses on tumor exposure of unconjugated and total MMAE. Simulations were performed at different dose levels, ranging from 0.1 to $10 \mathrm{mg} / \mathrm{kg}$. The model predicted (Supplemental Fig. 4) sustained exposure and longer Tmax of free and total MMAE in the coculture tumors, which mainly stems from the time taken by N87 cells to process T-vc-MMAE and redistribution of MMAE to bystanding cells.

Simulation of Intracellular Tubulin Occupancy for Each Cell Type in the Heterogeneous Tumor. Figure 4A shows simulated percentage tubulin occupancy by MMAE (Occ $\mathrm{Tub}_{\mathrm{T}}^{\mathrm{i}}$ ) for N87 and GFP-MCF7 cells in the 50:50 coculture tumor. Simulations were performed at $0.1,1$, and $10 \mathrm{mg} / \mathrm{kg}$ doses of T-vc-MMAE. It was observed that at lower doses, the difference in tubulin occupancy between HER2-high N87 cell and HER2-low GFP-MCF7 cell was much higher, which is expected, as most of the ADC molecules will gravitate toward Ag-positive cells at this dose level. As the dose increased, both the cells achieved very similar occupancy, which may be due to the saturation of Ag-mediated pathway. These simulations suggest that at lower doses, T-vc-MMAE may preferentially kill N87 cells over GFP-MCF7 cells. Figure 4B shows simulated tubulin occupancy for GFP-MCF7 cells with increasing fraction of $\mathrm{N} 87$ cells in the coculture tumor. It was observed that irrespective of the $\mathrm{ADC}$ dose, an increase in the fraction of N87 cells led to higher tubulin occupancy for GFP-MCF7 cells, which is consistent with the bystander effect phenomenon. Interestingly, it was observed that the improvement in tubulin occupancy was more prominent for $1 \mathrm{mg} / \mathrm{kg}$ dose compared with 0.1 or $10 \mathrm{mg} / \mathrm{kg}$ doses. This could be because at lower doses, the availability of MMAE may be limiting, and at higher doses, tubulin may be close to saturation. As such, these simulations suggest that there could be a bell-shaped relationship between ADC dose and the beneficial effects of the bystander effect.

Characterization of TGI Data Using the Systems PK-PD Model. Figure 6 shows individual model fittings for representative animals, whereas the fittings for all the animals along with goodness-of-fit (GoF) plots and visual
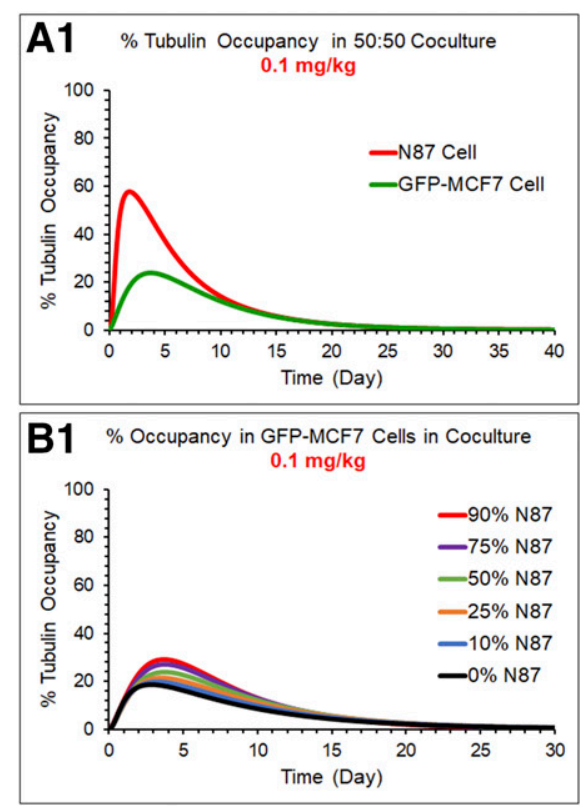

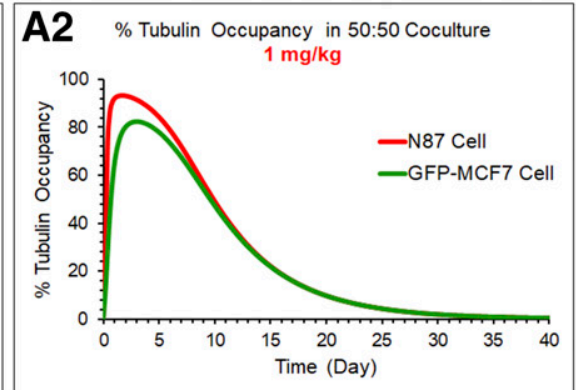

B2 \% Occupancy in GFP-MCF7 Cells in Coculture

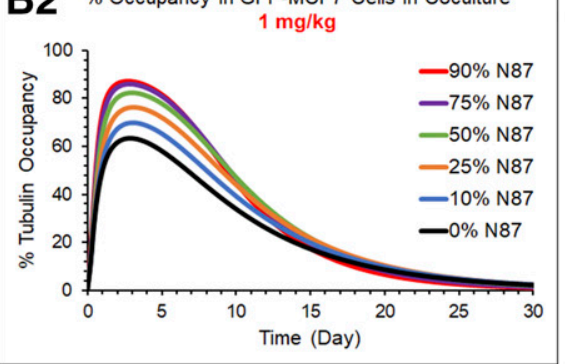

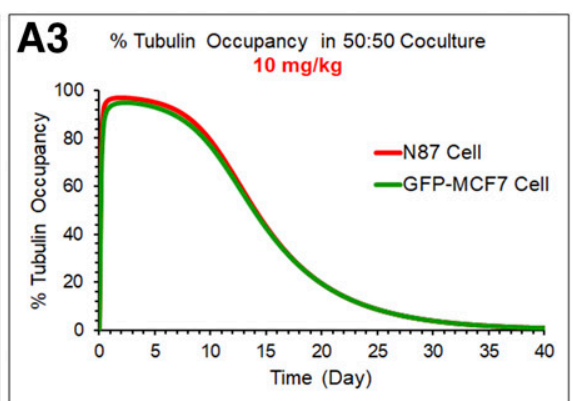

B3 \% Occupancy in GFP-MCF7 Cells in Coculture

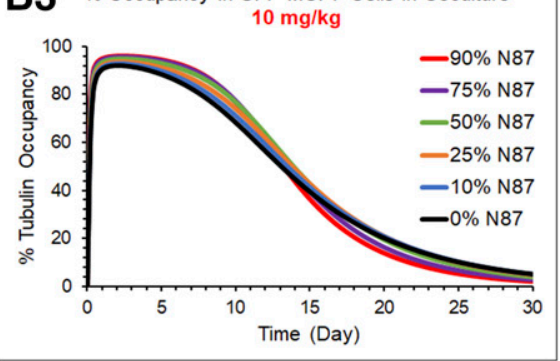

Fig. 4. (A) Simulations for intracellular occupancy of tubulin by MMAE (Occ ${ }_{\mathrm{Tub}}$ ) for GFP-MCF7 (profiles in green) and N87 (profiles in red) cells in the 50:50 coculture tumor after single dose of $0.1,1$, and $10 \mathrm{mg} / \mathrm{kg}$ i.v. T-vc-MMAE. (B) Simulations for improvement in tubulin occupancy of GFP-MCF7 cell (Occ ${ }_{\text {Tub }}^{\text {MCF }}$ ) after increasing percentages of N87 cell fraction within the heterogeneous tumor. Simulations were performed at $0.1,1$, and $10 \mathrm{mg} / \mathrm{kg}$ dose of T-vc-MMAE. 


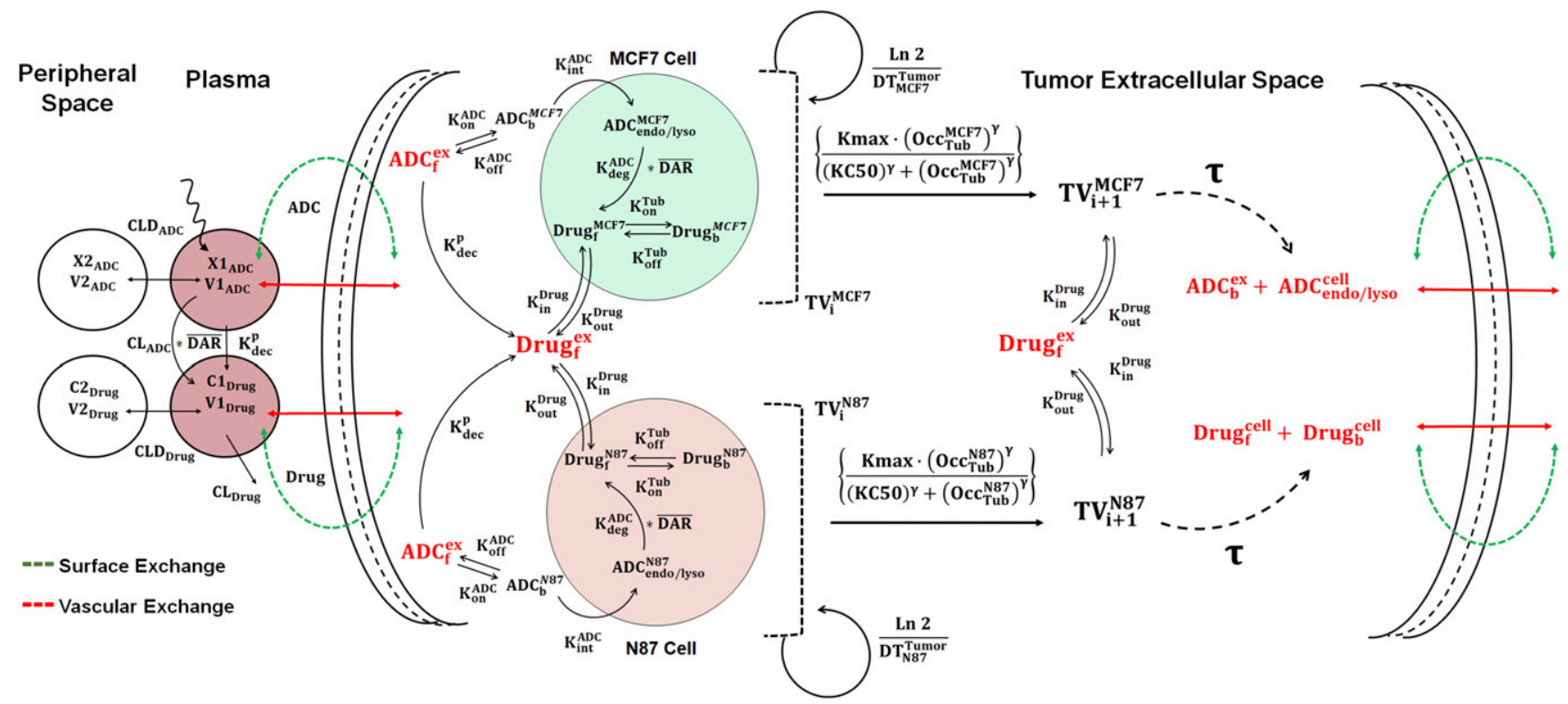

Fig. 5. Schematic diagram of the full systems PK-PD model developed to characterize bystander effect of T-vc-MMAE in a heterogeneous tumor containing N87 and GFP-MCF7 cells. The plasma and tumor pharmacokinetics, as well as disposition of T-vc-MMAE in each cell-type, is similar to Fig. 3. Intracellular occupancy of tubulin by MMAE $\left(\mathrm{Occ}_{\mathrm{Tub}}\right)$ in each cell type was assumed to drive the efficacy of ADC, which was characterized by shuttling of growing cells into nongrowing phases destined to die. It was assumed that growing and nongrowing cells contribute toward the disposition of T-vc-MMAE and free MMAE. When the cells die, intracellular content was assumed to release in tumor extracellular space and allowed to freely exchange with other living cells or systemic circulation.

predictive checks are provided in Supplemental Data (Supplemental Figs. 5-13). The population PK-PD model was able to simultaneously characterize all the TGI data reasonably well, as highlighted by the GoF plots (Supplemental Figs. 10 and 11) as well as prediction-corrected visual predictive checks (Supplemental Fig. 13). The doubling times for N87 and GFPMCF7 tumors were estimated with good precision and were found to be 13.6 and 9.1 days, respectively, with $\sim 30 \%$ intersubject variability on each parameter (Table 1). The efficacy for each cell type was driven using a killing function, which used common PD parameters (Kmax, KC50, $\tau$ ) to induce intracellular tubulin occupancy mediated cell cytotoxicity. Model-estimated in vivo Kmax and KC50 values of $0.4 \frac{1}{\text { day }}$ and $99.8 \%$ were very similar to in vitro estimated vales for MMAE-induced cell killing (Kmax of $0.72 \frac{1}{\text { day }}$ and KC50 of 98.3\% for N87 cells and 96.1\% for GFP-MCF7 cells) (Singh and Shah, 2019).

Simulations for Growth Profiles of N87 and GFP-MCF7 Tumor Fractions. The final PK-PD model was used to investigate the dynamics of N87 and GFPMCF7 tumor fractions within the coculture tumor, after treatment with different doses of T-vc-MMAE. It was observed (Supplemental Fig. 11) that in the beginning, the whole tumor declines in parallel to a decline in N87 and GFP-MCF7 fractions of the tumor. However, when the tumor starts to regrow, the majority of tumor fraction is composed of GFPMCF7 cells, which are less responsive to the ADC and shows a faster growth pattern.

Simulations to Investigate the Effect of Fractionated Dosing Regimen on Bystander Effect of ADC in a Heterogeneous Tumor. These simulations were performed to investigate how the rate and extent of bystander effect changes with varying ADC dose-levels, dosing regimens, and compositions of the heterogeneous tumors. Figure 7 provides the results from these simulations in the form of "MCF7 fraction" tumor volume changes with time. In the single-dose group, it was observed that an increase in percent of N87 fraction in the heterogeneous tumor led to higher killing of MCF7 cells, especially at the lower dose. This observation is consistent with the expected enhancement of $\mathrm{ADC}$ bystander effect with an increase in the fraction of Agpositive cells. When the ADC doses were fractionated, it was observed that fractionated dosing regimen was more efficacious in killing $100 \% \mathrm{MCF}$ tumors and "MCF7 fractions" in the coculture tumors. This effect was also more prominent at lower dose levels of ADC. These simulations suggest that fractionated dosing regimen maybe more beneficial in achieving sustaining tubulin occupancy within Ag-negative tumor cells. In addition, it was observed that as the percent of N87 fraction increased beyond a certain level (i.e., 50\%), there was no further improvement in the bystander killing by the ADC, even in the presence of the fractionated dosing regimen.

Within the simulation results (Supplemental Figs. 14 and 15) for the fractionated dosing regimen in the form of "total" tumor volume (along with each cell-type fraction) changes with time, it was observed that in comparison with the single dose, the fractionated dosing regimen leads to an improvement in the efficacy of ADC for the heterogeneous tumor. The majority of this improvement stemmed from enhanced killing of N87 tumor fraction, which in turn contributed to higher killing of GFP-MCF7 fraction of the tumor via the bystander effect.

\section{Discussion}

A systems PK-PD model that can characterize in vivo bystander effect of ADCs in heterogeneous solid tumors could significantly improve the discovery and development of 
Control Group
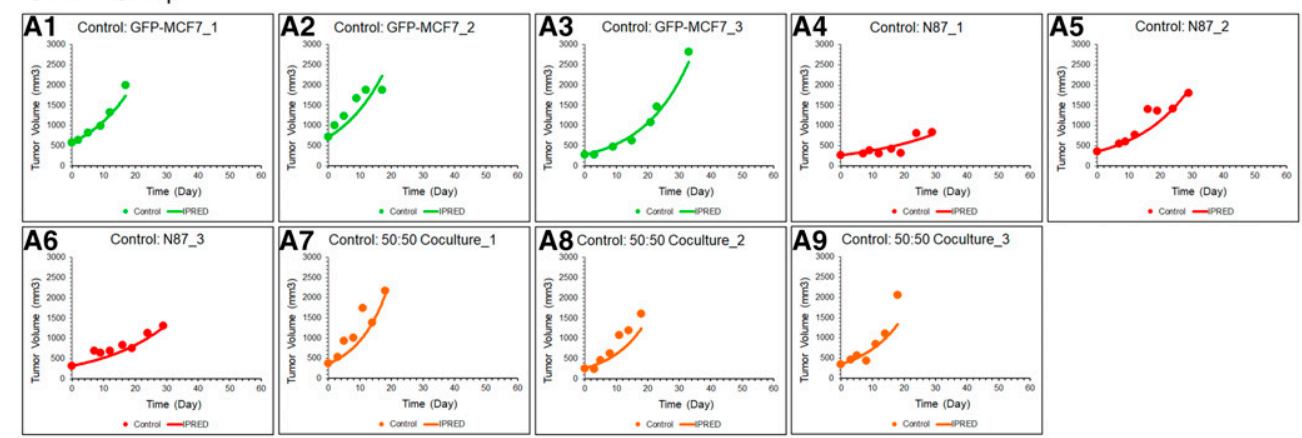

Treatment Group: $1 \mathrm{mg} / \mathrm{Kg}$
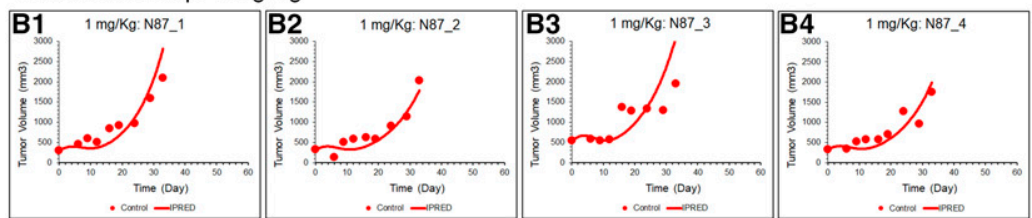

Treatment Group: $3 \mathrm{mg} / \mathrm{Kg}$
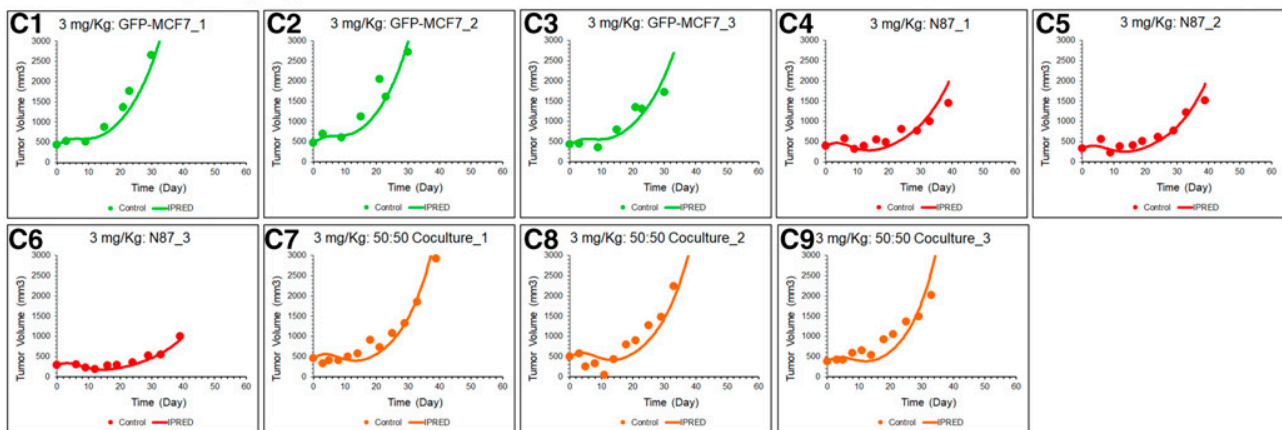

Treatment Group: $5 \mathrm{mg} / \mathrm{Kg}$
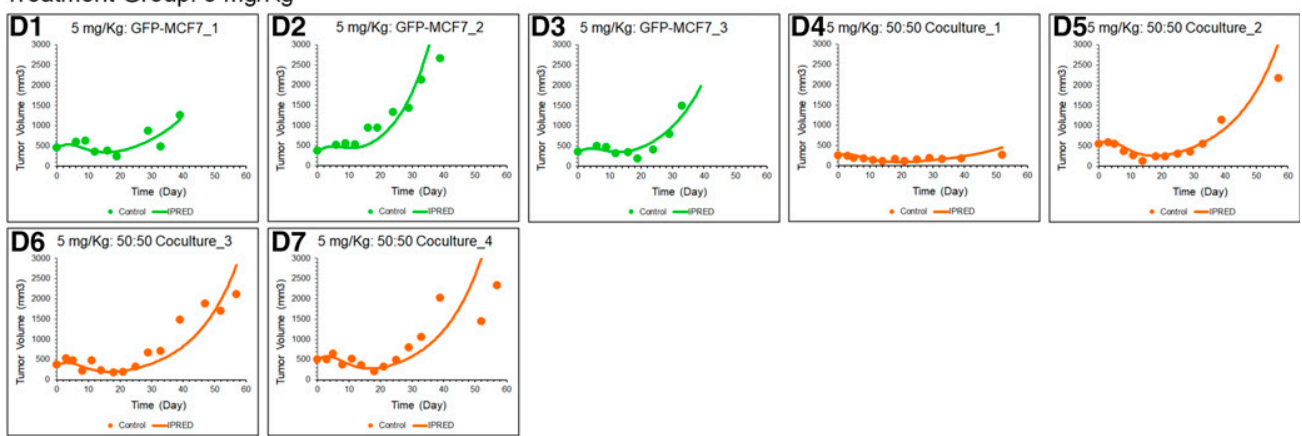

Treatment Group: $10 \mathrm{mg} / \mathrm{Kg}$
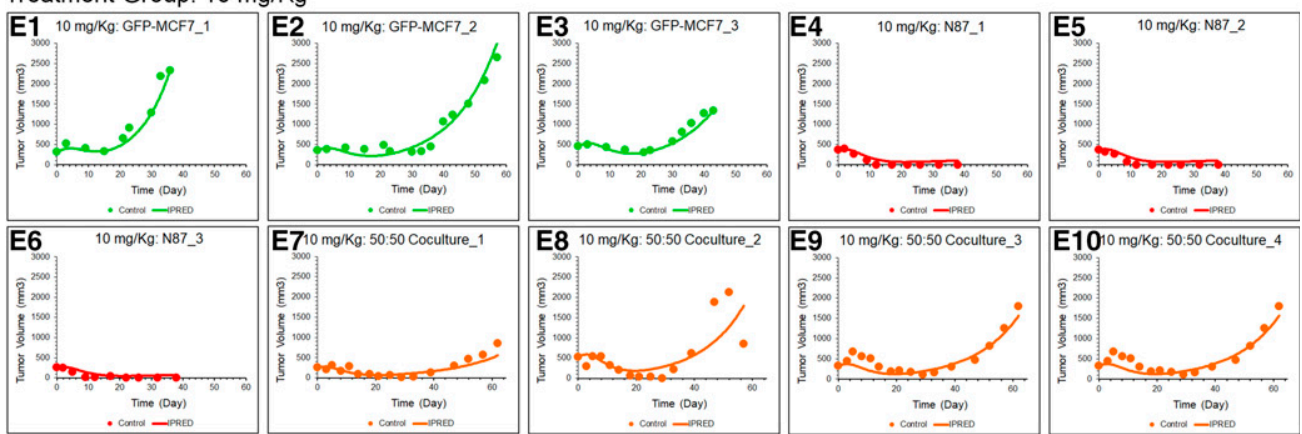

Fig. 6. Representative observed and model fitted tumor growth profiles for GFP-MCF7 (green), N87 (red), and coculture (orange) tumors. (A) Control group, (B) $1 \mathrm{mg} / \mathrm{kg}$, (C) $3 \mathrm{mg} / \mathrm{kg}$, (D) $5 \mathrm{mg} / \mathrm{kg}$, and (E) $10 \mathrm{mg} / \mathrm{kg} \mathrm{T}-\mathrm{vc}-$ MMAE. Individual fits for all animals as well as GoF plots (Supplemental Figs. 5-10). 
clinically successful ADCs. Toward this goal we have taken a stepwise approach of conducting experiments and building systems models (Supplemental Fig. 2). Current investigation represents the final step of this process that requires in vivo experiments in a heterogeneous tumor model. The heterogeneous tumor model was developed by inoculating a mixture of GFP-MCF7 and N87 cells in the mice. Because both the cells have different growth rates, after several attempts, a ratio of $\sim 1$ million GFP-MCF7 and $\sim 9$ million N87 cells was finalized to obtain a coculture composition of 50:50 at tumor volumes $\sim 350-400 \mathrm{~mm}^{3}$. This coculture composition was confirmed by semiquantitative IHC analysis (Fig. 1).

A detailed PK and TGI study was conducted on the heterogeneous tumors using our tool ADC, T-vc-MMAE (Fig. 2). PK-PD data generated using the heterogeneous tumor-bearing mice were also compared with the data from $100 \%$ N87 and GFP-MCF7 tumor-bearing mice. It was observed that there was no difference in the plasma PK of different ADC analytes between the three tumor models. This result suggests that presence of tumor did not significantly affect systemic PK of ADC and confirms our previous observation that the amount of payload coming from the tumor space does not contribute significantly toward overall systemic PK of the drug (Shah et al., 2014). Tumor PK data revealed that coculture tumors had prolonged exposure of free and total MMAE compared with GFP-MCF7 and N87 tumors. We hypothesize that this may stem from the fact that it takes time for $\mathrm{N} 87(\mathrm{Ag}+)$ cells to first process the ADC and release the free MMAE in the tumor extracellular space, which subsequently redistributes and accumulates in the bystanding GFP-MCF7 (Ag-) cells.

Tumor growth data revealed that 50:50 coculture tumors grew at a rate similar to the fast-growing GFP-MCF7 tumors, suggesting faster growing cells within a heterogeneous tumor will eventually take over most of the tumor in the absence of any treatment. TGI data revealed that the efficacy of $\mathrm{ADC}$ in 50:50 coculture tumors was always higher than the GFP-MCF7 tumors, and this enhanced efficacy persisted unless there was a regrowth following ablation of N87 cells. This observation accentuated the fact that killing of N87 cells was important to exert the bystander effect in GFP-MCF7 cells (Singh et al., 2016b); however, once the N87 cells are ablated, there won't be any bystander effect, and the coculture tumors will start growing at the rate similar to GFP-MCF7 tumors. As such, an ideal ADC and dosing regimen should lead to a parallel decline in antigen-positive and antigen-negative cells within a heterogeneous tumor.

To develop the systems PK-PD model for ADC, first our previously published tumor PK model was expanded to include two different populations of cells growing at distinct growth rates. All the parameters associated with plasma PK, tumor distribution, and cellular PK of ADC in each cell type were fixed to our previously reported values. The final tumor disposition model (Fig. 3) was able to a priori predict tumor PK of all ADC analytes. The PK model also successfully predicted delayed Tmax associated with the exposure of total and free MMAE in the coculture tumors compared with N87 and GFP-MCF7 tumors (Supplemental Fig. 4). The model was also used to simulate tubulin occupancy in different cell types within the heterogeneous tumor at different dose levels (Fig. 4). It was observed that the difference in tubulin occupancy between the two cells was more pronounced at lower doses, where N87 cells showed higher occupancy compared with GFP-MCF7 cells. These simulations corroborated TGI data, in which higher ADC efficacy was observed for N87 tumors compared with GFP-MCF7 tumors, and also supported our approach of utilizing tubulin occupancy by MMAE as a driver for ADC efficacy (Singh and Shah, 2019). The effect of heterogeneous tumor composition on tubulin occupancy was also simulated using the PK model. It was found that with increasing fraction of N87 cells in the tumor, there was an increase in tubulin occupancy of GFP-MCF7 cells, which was consistent with the expectation of ADC bystander effect. However, simulations revealed that the extent of improvement in tubulin occupancy of GFP-MCF7 cells was dose-dependent, and there may be a bell-shaped relationship between $\mathrm{ADC}$ dose and the improvement in bystander effect following enhancement of Ag+ cell fraction in a heterogeneous tumor. However, this hypothesis needs to be validated using experimental data.

The heterogeneous tumor PK model was integrated with a PD model to make the final systems PK-PD model (Fig. 5), which used intracellular occupancy of tubulin as the driver of ADC efficacy. The final model was able to simultaneously characterize all the TGI data reasonably well, using a single set of $\mathrm{PD}\left(K_{\max }, K C_{50}, \tau\right)$ parameters. In addition, the parameter estimates obtained from in vivo efficacy studies were very similar to previously obtained parameters from in vitro estimates (Singh and Shah, 2019). This observation highlights the importance of adopting a systems approach and suggests a good correlation between in vitro and in vivo efficacy of ADCs. The system PK-PD model was used to investigate the effect of ADC dose and increasing percentage of Ag+ cell fraction on the growth of $\mathrm{Ag}-$ cell fraction. It was observed that the bystander effect of ADC was most optimal at $1-5 \mathrm{mg} / \mathrm{kg}$ doses, beyond which the effect seemed to saturate. Additionally, it was observed that having a certain fraction of $\mathrm{Ag}+$ cells (e.g., 50\%) within the heterogeneous tumor would be enough to achieve the maximum bystander effect of ADC. The PK-PD model was also used to investigate the effect of fractionated dosing regimen on the efficacy of $\mathrm{ADC}$ in a heterogeneous tumor (Figs. 7 and 8). Model simulations revealed that dose fractionation may further improve overall efficacy and bystander effect of T-vc-MMAE, as it will prolong the duration of tubulin occupancy in each cell type. While this is an exciting finding, it needs to be corroborated by experimental results, and the readers should be cautious before generalizing it to other ADCs and tumors. Nonetheless, the systems PK-PD model presented here for characterizing in vivo bystander effect of ADC in a heterogeneous tumor remains generalizable.

In summary, here we have presented a novel systems PK$\mathrm{PD}$ model for ADCs that accounts for multiple populations of tumor cells. The model could account for differential exposures of ADC in each cell type and can simultaneously characterize ADC-induced direct killing and bystander effect. Because the modeling paradigm presented here is formulated on a singlecell level, it could also be extended to other therapeutic scenarios in which a molecule interacts with multiple cell types within a tumor microenvironment (e.g., bispecifics interacting with tumor and immune cells). Going forward, we plan to translate this systems PK-PD to predict bystander 


\section{Single Dosing Regimen}
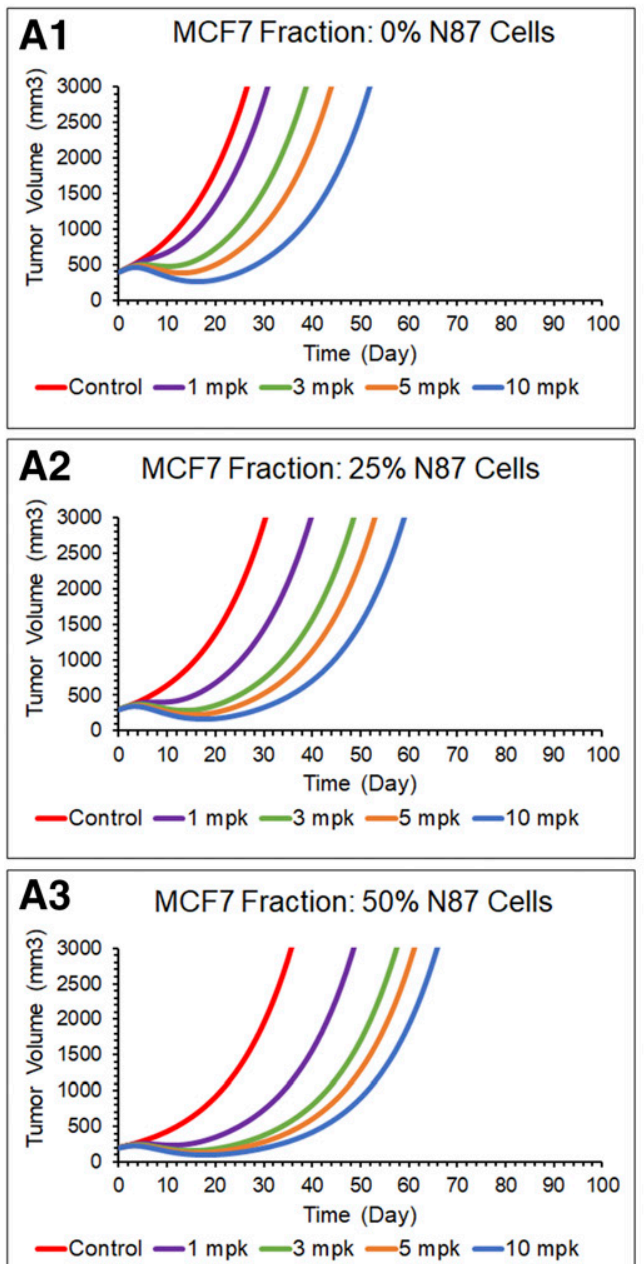

A4 MCF7 Fraction: 75\% N87 Cells
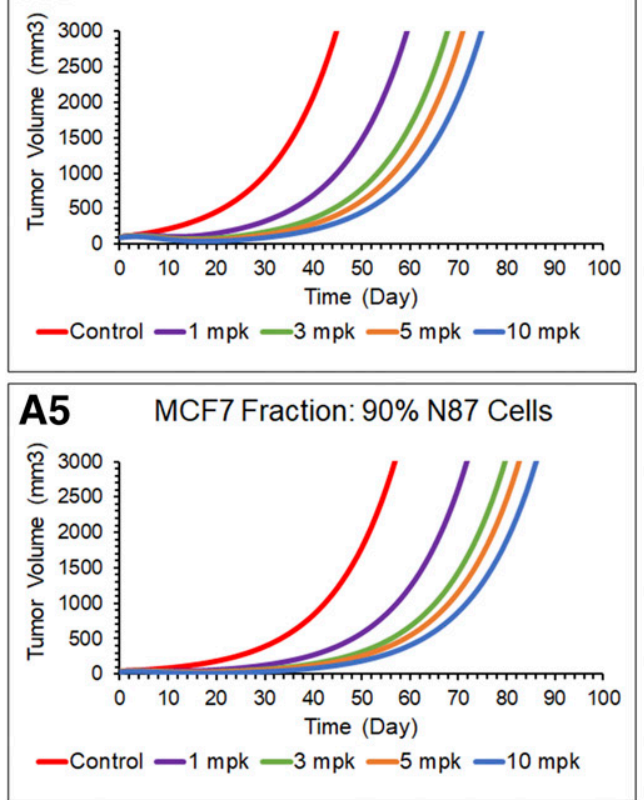

\section{Fractionated Dosing Regimen}
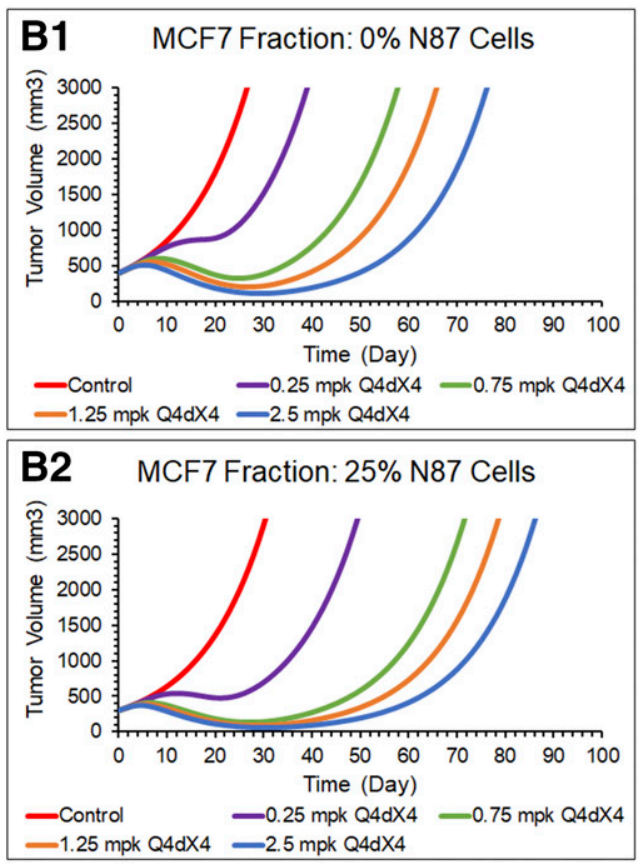

B3 MCF7 Fraction: $50 \%$ N87 Cells

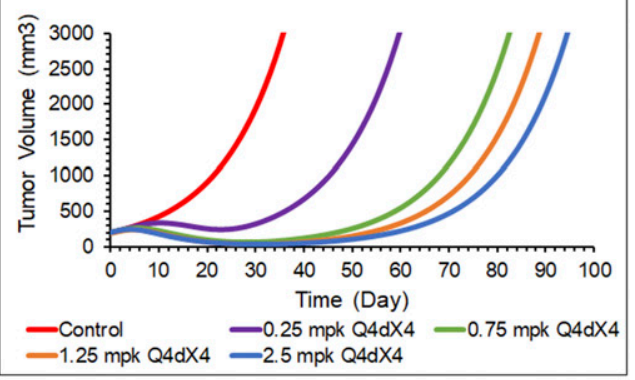

\section{B4 MCF7 Fraction: 75\% N87 Cells}

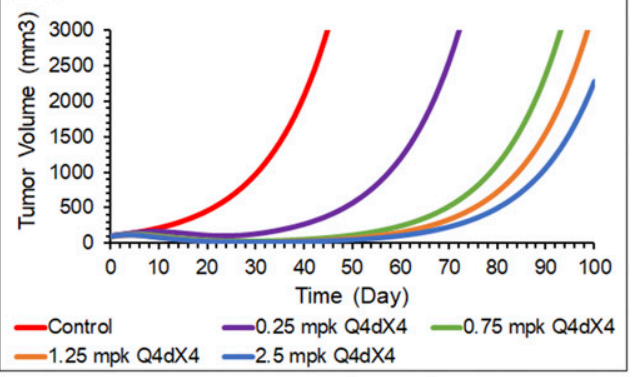

B5 MCF7 Fraction: $90 \%$ N87 Cells

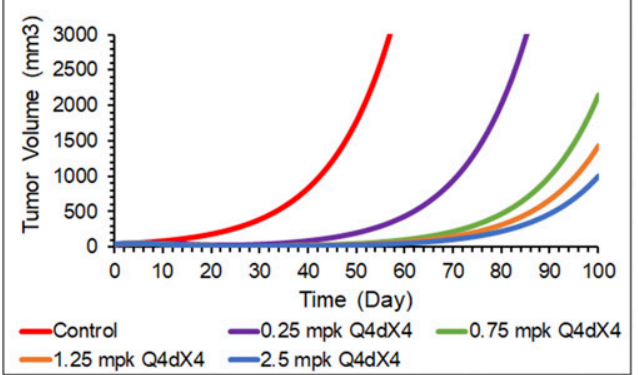

Fig. 7. Simulations for the growth of "MCF7 fraction" of a coculture tumor with varying percentage of N87 cells. Four different dose levels of T-vcMMAE were evaluated: $1,3,5$, and $10 \mathrm{mg} / \mathrm{kg}$, administered either as a single dose (left panels) or fractionated into four equal doses administered every 4 days (Q4dX4, right panels). (A1 and B1) $0 \%$ N87, (A2 and B2) $25 \%$ N87, (A3 and B3) $50 \%$ N87, (A4 and B4) 75\% N87, and (A5 and B5) 90\% N87 cells. 

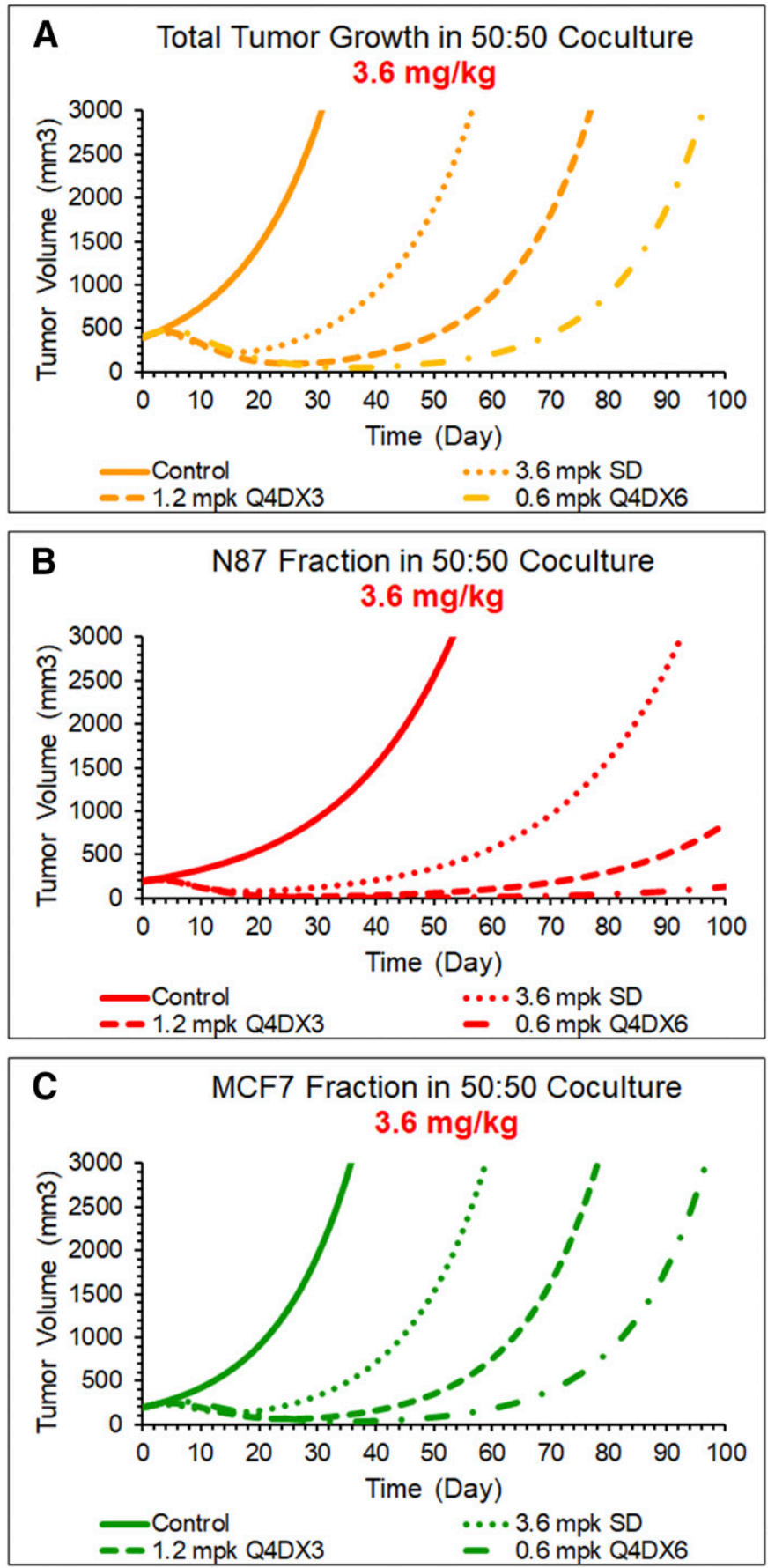

Fig. 8. Comparative evaluation of tumor growth inhibition (TGI) of (A) 50: 50 coculture tumor, (B) N87 fraction of the tumor, or (C) GFP-MCF7 fraction of the tumor, after single $3.6 \mathrm{mg} / \mathrm{kg}$ dose of $\mathrm{ADC}$ or fractionated dosing regimens of $1.2 \mathrm{mg} / \mathrm{kg}$ given Q4dX3 or $0.6 \mathrm{mg} / \mathrm{kg} \mathrm{Q} 4 \mathrm{dX} 6$.

effect of ADCs in the clinic (Shah et al., 2012; Betts et al., 2016; Singh and Shah, 2017a).

\section{Acknowledgments}

The authors would like to acknowledge Dr. Sharad Sharma and Shabkaiz Masih for their help in development of analytical techniques and establishing tumor models. The authors would also like to acknowledge Dr. Wade Sigurdson from the School of Medicine and Biomedical Sciences, University at Buffalo, for his helpful assistance with fluorescent microscopy imaging of tumor samples. The authors would also like to acknowledge Zhe $\mathrm{Li}$ and David Bussing for their helpful assistance when needed while executing certain experiments.

\section{Authorship Contributions}

Participated in research design: Singh, Shah.

Conducted experiments: Singh, Seigel, Guo, Verma, Wong, Cheng.

Performed data analysis: Singh, Seigel, Shah.

Wrote or contributed to the writing of the manuscript: Singh, Shah.

\section{References}

Anthony LKR (2018) Review of the ADC clinical pipeline, in World ADC Summit, Berlin.

Betts AM, Haddish-Berhane N, Tolsma J, Jasper P, King LE, Sun Y, Chakrapani S, Shor B, Boni J, and Johnson TR (2016) Preclinical to clinical translation of antibody-drug conjugates using PK/PD modeling: a retrospective analysis of inotuzumab ozogamicin. AAPS $J$ 18:1101-1116.

Cilliers C, Guo H, Liao J, Christodolu N, and Thurber GM (2016) Multiscale modeling of antibody-drug conjugates: connecting tissue and cellular distribution to whole animal pharmacokinetics and potential implications for efficacy. AAPS $J \mathbf{1 8}$ $1117-1130$

Del Monte U (2009) Does the cell number $10\left({ }^{9}\right)$ still really fit one gram of tumor tissue? Cell Cycle 8:505-506.

Donoghue JF, McGavigan CJ, Lederman FL, Cann LM, Fu L, Dimitriadis E, Girling JE, and Rogers PA (2012) Dilated thin-walled blood and lymphatic vessels in human endometrium: a potential role for VEGF-D in progestin-induced breakthrough bleeding. PLoS One 7:e30916.

Khot A, Sharma S, and Shah DK (2015) Integration of bioanalytical measurements using PK-PD modeling and simulation: implications for antibody-drug conjugate development. Bioanalysis 7:1633-1648.

Kovtun YV, Audette CA, Ye Y, Xie H, Ruberti MF, Phinney SJ, Leece BA, Chittenden T, Blättler WA, and Goldmacher VS (2006) Antibody-drug conjugates designed to eradicate tumors with homogeneous and heterogeneous expression of the target antigen. Cancer Res 66:3214-3221.

Lambert JM (2015) Antibody-drug conjugates (ADCs): magic bullets at last!. Mol Pharm 12:1701-1702.

Lavielle M and Mentré F (2007) Estimation of population pharmacokinetic parameters of saquinavir in HIV patients with the MONOLIX software. J Pharmacokinet Pharmacodyn 34:229-249.

Mantaj J, Jackson PJ, Rahman KM, and Thurston DE (2017) From anthramycin to pyrrolobenzodiazepine (PBD)-containing antibody-drug conjugates (ADCs). Angew Chem Int Ed Engl 56:462-488.

Ogitani Y, Hagihara K, Oitate M, Naito H, and Agatsuma T (2016) Bystander killing effect of DS-8201a, a novel anti-human epidermal growth factor receptor 2 antibody-drug conjugate, in tumors with human epidermal growth factor receptor 2 heterogeneity. Cancer Sci 107:1039-1046.

Schneider CA, Rasband WS, and Eliceiri KW (2012) NIH Image to ImageJ: 25 years of image analysis. Nat Methods 9:671-675.

Seol H, Lee HJ, Choi Y, Lee HE, Kim YJ, Kim JH, Kang E, Kim SW, and Park SY (2012) Intratumoral heterogeneity of HER2 gene amplification in breast cancer: its clinicopathological significance. Mod Pathol 25:938-948.

Shah DK, Haddish-Berhane N, and Betts A (2012) Bench to bedside translation of antibody drug conjugates using a multiscale mechanistic PK/PD model: a case study with brentuximab-vedotin. $J$ Pharmacokinet Pharmacodyn 39: $643-659$

Shah DK, King LE, Han X, Wentland JA, Zhang Y, Lucas J, Haddish-Berhane N, Betts A, and Leal M (2014) A priori prediction of tumor payload concentrations: preclinical case study with an auristatin-based anti-5T4 antibody-drug conjugate. AAPS J 16:452-463.

Singh AP, Guo L, Verma A, Wong GG, and Shah DK (2019) A cell-level systems PK$\mathrm{PD}$ model to characterize in vivo efficacy of ADCs. Pharmaceutics 11.

Singh AP, Krzyzanski W, Martin SW, Weber G, Betts A, Ahmad A, Abraham A Zutshi A, Lin J, and Singh P (2015a) Quantitative prediction of human pharmacokinetics for mAbs exhibiting target-mediated disposition. AAPS $J \mathbf{1 7}$ : 389-399.

Singh AP, Maass KF, Betts AM, Wittrup KD, Kulkarni C, King LE, Khot A, and Shah DK (2016a) Evolution of antibody-drug conjugate tumor disposition model to predict preclinical tumor pharmacokinetics of trastuzumab-emtansine (T-DM1). AAPS $J$ 18:861-875.

Singh AP and Shah DK (2017a) Application of a PK-PD modeling and simulationbased strategy for clinical translation of antibody-drug conjugates: a case study with trastuzumab emtansine (T-DM1). AAPS J 19:1054-1070.

Singh AP and Shah DK (2017b) Measurement and mathematical characterization of cell-level pharmacokinetics of antibody-drug conjugates: a case study with trastuzumab-vc-MMAE. Drug Metab Dispos 45:1120-1132.

Singh AP and Shah DK (2018) Utility of PK-PD modeling and simulation to improve decision making for antibody-drug conjugate development, in Innovations for NextGeneration Antibody-Drug Conjugates Cancer Drug Discovery and Development (Damelin M, Humana Press, Cham.

Singh AP and Shah DK (2019) A "dual" cell-level systems PK-PD model to characterize the bystander effect of ADC. J Pharm Sci 108:2465-2475.

Singh AP, Sharma S, and Shah DK (2016b) Quantitative characterization of in vitro bystander effect of antibody-drug conjugates. $J$ Pharmacokinet Pharmacodyn 43: 567-582.

Singh AP, Shin YG, and Shah DK (2015b) Application of pharmacokineticpharmacodynamic modeling and simulation for antibody-drug conjugate development. Pharm Res 32:3508-3525. 
Thurber GM, Schmidt MM, and Wittrup KD (2008a) Antibody tumor penetration: transport opposed by systemic and antigen-mediated clearance. Adv Drug Deliv Rev 60:1421-1434.

Thurber GM, Schmidt MM, and Wittrup KD (2008b) Factors determining antibody distribution in tumors. Trends Pharmacol Sci 29:57-61.

van Geel R, Wijdeven MA, Heesbeen R, Verkade JM, Wasiel AA, van Berkel SS, and van Delft FL (2015) Chemoenzymatic conjugation of toxic payloads to the globally conserved N-glycan of native mAbs provides homogeneous and highly efficacious antibody-drug conjugates. Bioconjug Chem 26:2233-2242.

Yang J, Mager DE, and Straubinger RM (2010) Comparison of two pharmacodynamic transduction models for the analysis of tumor therapeutic responses in model systems. AAPS J 12:1-10.
Address correspondence to: Dr. Dhaval K. Shah, Department of Pharmaceutical Sciences, School of Pharmacy and Pharmaceutical Sciences, University at Buffalo, The State University of New York, 455 Pharmacy Building, Buffalo, NY 14214-8033. E-mail: dshah4@buffalo.edu; or Dr. Aman P. Singh, Department of Pharmaceutical Sciences, School of Pharmacy and Pharmaceutical Sciences, University at Buffalo, The State University of New York, 455 Pharmacy Building, Buffalo, NY 14214-8033. E-mail: amanpree@buffalo.edu, Discovery and Translational Research, Biologics Development Sciences, Janssen Biotherapeutics, 1400 McKean Road, Spring House, PA 19002. E-mail: ASing215@its.jnj.com 